\title{
Effect of void arrangement on ductile damage mechanisms in nodular graphite cast iron: in situ 3D measurements
}

\author{
Ante Buljac ${ }^{\mathrm{a}, \mathrm{b}}$, Lukas Helfen ${ }^{\mathrm{c}, \mathrm{d}}$, François Hild ${ }^{\mathrm{a}}$, Thilo F. Morgeneyer ${ }^{\mathrm{b}, *}$ \\ ${ }^{a}$ Laboratoire de Mécanique et Technologie (LMT), ENS Paris-Saclay, CNRS, \\ Université Paris-Saclay, 61 avenue du Président Wilson, 94235 Cachan, France \\ ${ }^{b}$ MINES ParisTech, PSL Research University, Centre des Matériaux, CNRS UMR 7633, \\ BP 87, 91003 Evry, France \\ ${ }^{c}$ Institute for Photon Science and Synchrotron Radiation \\ Karlsruhe Institute of Technology (KIT), 76131 Karlsruhe, Germany \\ ${ }^{d}$ European Synchrotron Radiation Facility (ESRF), 38043 Grenoble, France
}

\section{Abstract}

The effect of the arrangement of two artificial voids on void growth and coalescence mechanisms is studied in flat specimens made of nodular graphite cast iron via in situ 3D laminography imaging and digital volume correlation for bulk displacement measurement. Two configurations of machined holes are studied i) $90^{\circ}$ with respect to the loading direction to mimic internal necking, and ii) $45^{\circ}$ to attempt to trigger a void sheeting mechanism between the artificial voids. The machined holes play the role of primary voids, the graphite nodules that of secondary voids. At a lower scale there is a smaller third population of voids. The $45^{\circ}$ void orientation successfully causes a shear band between the primary voids. However, the growth and co-

\footnotetext{
${ }^{*}$ Corresponding author.

Email address: thilo.morgeneyer@ensmp.fr (Thilo F. Morgeneyer)
} 
alescence mechanisms of voids from graphite nodules are similar for the two configurations. Internal necking is found for nodules that are close to each other and void sheeting for large ferrite ligaments between nodules. Strain fields in the region between machined holes show necking for both cases as well as volume increase, i.e., positive trace of the strain tensor, confirmed by image analysis. The strain to failure in this inter-void region is evaluated in the bulk and also locally, between nodules and for different coalescence configurations. Similar levels are observed in both cases.

Keywords: Cast iron, Digital Volume Correlation (DVC), Ductile damage, Multiscale analysis, Laminography

\section{Introduction and motivation}

A whole range of engineering materials has been optimized throughout history following the idea of avoiding brittle fracture. This quest resulted in significant portion of modern materials failing in ductile manners. The constant quest for more fuel efficient and hence lighter design implies extended knowledge about the ductile damage mechanisms during forming and inservice conditions. Significant efforts in modelling of ductile failure processes have been made during the past few decades which resulted in substantial progress of knowledge [4]. However, the full understanding of ductile failure mechanisms under specific stress states still remains an open question.

Low stress triaxialities $(<1)$ induce phenomena that cannot be handled with classical ductile damage models $[17,32]$ that are tailored for higher triax- 
ialities. Phenomenological approaches where the strain to failure $\epsilon_{f}$ is defined as a function of stress triaxiality $\eta$ and Lode parameter $\mu[33,1,16,52]$ yield satisfactory results in industrial contexts but still without general applicability. There is no robust ductile damage model that is able to correctly predict fracture loci $\epsilon_{f}(\eta, \mu)$ under arbitrary loading paths [13, 12]. It has been demonstrated in previous works that both damage growth [37] and strain to failure values [3] are loading path dependent, i.e., nonproportional loading conditions lead to different $\epsilon_{f}$ values for similar stress-averaged triaxialities.

The main reason for the above mentioned shortcomings is an incomplete understanding of ductile damage mechanisms. The lack of quantitative experimental data and their questionable interpretation result in case-sensitive model calibrations where the underlying physics is not fully captured.

3D imaging methods, e.g., Synchrotron Radiation Computed Tomography (SRCT) [22, 21, 23, 29], allow for in-situ monitoring of the three principal stages of ductile damage, namely nucleation, growth and coalescence of the voids at the microscale. While tomography is limited to scanning stick-like samples with small cross sectional areas, Synchrotron Radiation Computed Laminography (SRCL) [19, 18, 25, 11, 36, 48] enables regions of interest (ROIs) in laterally extended objects to be scanned. In the context of mechanical experiments this feature facilitates performing in-situ experiments with sheet- or panel-like samples [54, 26] with more engineering relevant boundary conditions, different levels of stress triaxialities and significant sizes of plastic deformation zones. Digital Volume Correlation (DVC) can be employed to 
measure 3D bulk kinematic fields [43]. The measured displacement fields can be used for driving FE simulations that account for underlying microstructures $[9,42]$. The proposed method where the FE model accounts for the real microstructure and is driven by measured boundary conditions can be extended to inverse identification purposes at the microstructure level.

In this work in-situ laminography experiments are performed on nodular graphite cast iron sheet samples that contain two machined holes. The geometry of the samples is inspired by the work of Weck et al. [51]. Two variants of the sample geometry will be studied, namely, a) two holes are machined at $45^{\circ}$, b) two holes are machined at $90^{\circ}$ with respect to the loading direction. The general idea is to induce natural localization between the machined holes and observe whether there are any fundamental differences between damage mechanisms in the localized band for the two cases. The material itself contains two particle populations, namely, graphite nodules considered as voids because of their weak mechanical properties $[15,53,6,20]$ and the population of small particles present in the ferritic matrix before loading. Hence, after machining holes with a diameter one order of magnitude higher than that of nodules, the proposed configuration presents a rather unique opportunity to observe three void populations in a single experiment.

The laminography technique enables us to focus on void growth in the sheet for the first time under such boundary conditions while DVC will be used for measuring the bulk kinematics. The estimated strain fields will be utilized for the determination of strains to failure for $90^{\circ}$ and $45^{\circ}$ configura- 
tions in the ligaments between the nodules and on the scale of the macroband between the machined holes. Void growth in the examined region and individual growth of the chosen voids will be reported by using segmentation technique on laminography images. Additionally, mean void growth measurements will be supported by analyzing the volumetric part of the strain tensor. Finally, measured meso- and micro-coalescence and fracture criteria in terms of both laminography and DVC kinematic data will be analyzed.

\section{Experimental methods}

\subsection{Sample preparation}

The studied material is a commercial nodular graphite cast iron with the serial code EN-GJS-400 [20]. The specimen geometry, which is shown in Figure 1, is inspired by the work of Weck et al. [51].

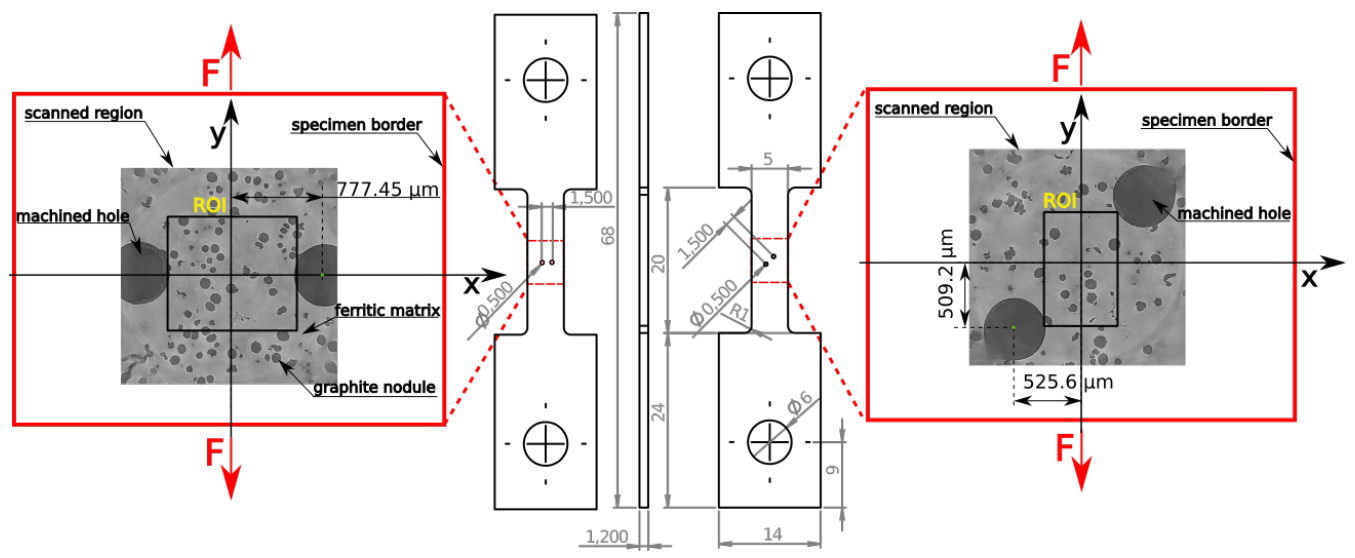

Figure 1: Drawings of the two samples with the scanned regions and the regions of interest (ROIs) for DVC analyses 
The holes $500 \mu \mathrm{m}$ in diameter have been machined by means of Electrical Discharge Machining (EDM). The nodules have a characteristic size of around $60 \mu \mathrm{m}$. Hence, they can be considered as distinct void populations with respect to the machined holes. In Ref. [51], the authors used machined holes of micrometer size. Consequently, the observation of the secondary void population was very limited due to the current imaging technique resolution [44]. Conversely, the larger size of the holes and graphite nodules in this work shall allow for such observations. Furthermore, the initial small graphite fragments in the ferritic matrix $(<1 \mu \mathrm{m})$ can also be detected in laminography scans, therefore they are considered as a third void population as illustrated in Figure 2(b).

\subsection{In-situ Laminography}

The load is manually adjusted via a displacement control in the testing device operated by screw rotation. After each load increment, a scan is acquired while the sample is rotated about the laminographic axis (i.e., approximately parallel to the specimen thickness direction). This axis is inclined with respect to the X-ray beam direction by an angle $\theta \approx 60^{\circ}$. The schematic view of the laminography setup together with the loading device are shown in Figure 2(a). The 3D images used in this work were obtained at beamline ID15A

of the European Synchrotron Radiation Facility (ESRF, Grenoble, France) with white beam of about $60 \mathrm{keV}$ average energy. Using 3,000 projection angles per scan, the series of radiographs is then processed with a filtered- 
back projection algorithm [31] to reconstruct 3D volumes. The parameter optimization has been performed automatically using a GPU-accelerated implementation [50]. For each scan, the reconstructed volume has a size of $1600 \times 1600 \times 1600$ voxels. The physical size (side length) of 1 cubic voxel is equal to $1.1 \mu \mathrm{m}$. The scanned regions are concentrated in the ligaments between and partly incorporate the two machined holes (Figure 1). Overall 24 scans were performed for the experiment with the $90^{\circ}$ configuration while 12 scans were acquired for the $45^{\circ}$ configuration.

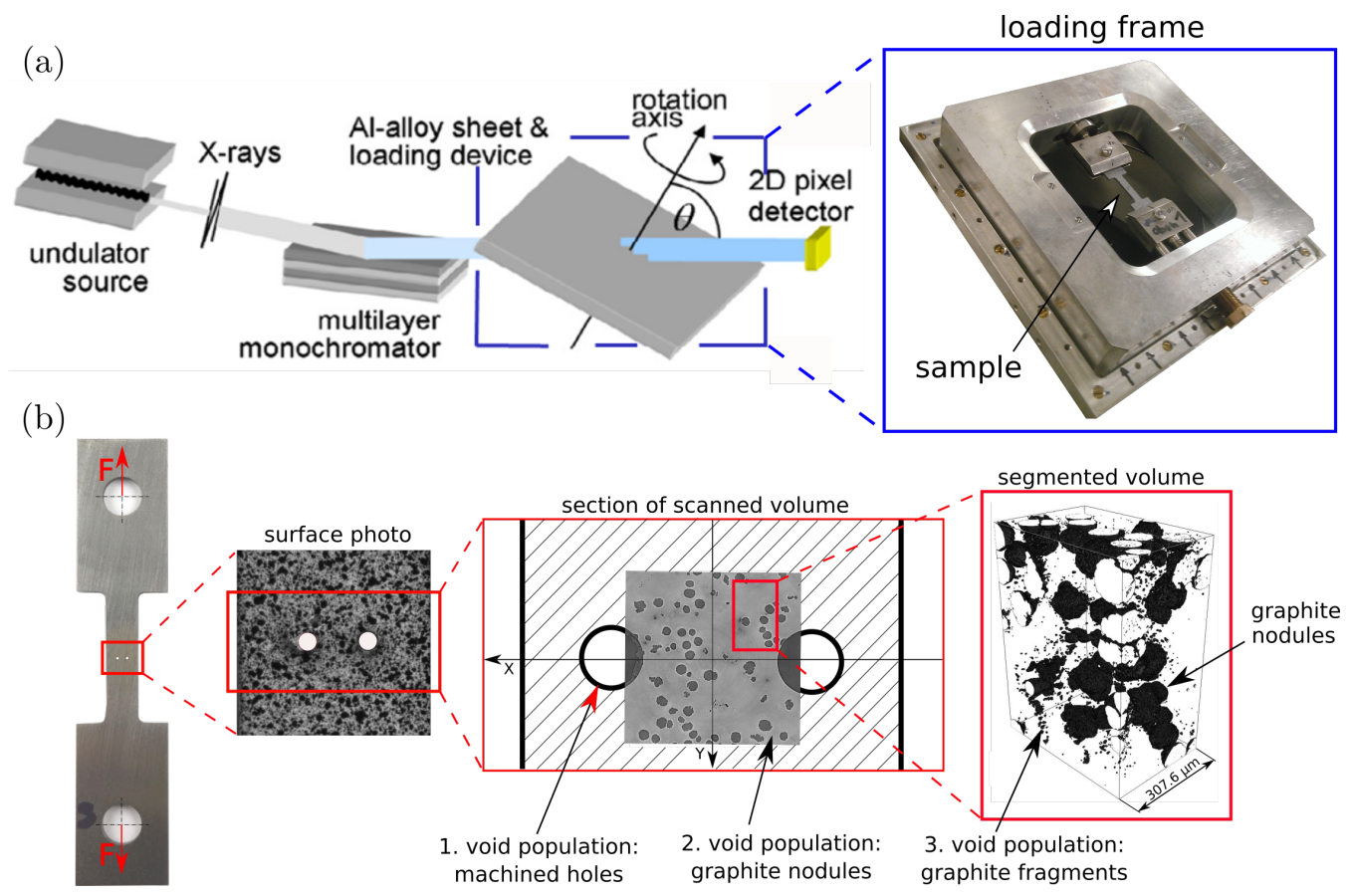

Figure 2: (a) Schematic view of laminography setup; (b) sample with scanned region: 3 void populations 


\subsection{Digital Volume Correlation}

The reconstructed volume is represented by a discrete gray level field (8bit deep) of the spatial (voxel) coordinate $\mathbf{x}$. Using the whole volume size for the correlation procedure would be computationally too demanding. Additionally, to cover larger regions of interest (ROIs) for the DVC calculations the original volumes were binned, i.e., blocks of 8 voxels are replaced with the corresponding supervoxel having the mean gray level of the block. Therefore an ROI of size $512 \times 544 \times 544$ supervoxels for the $90^{\circ}$ configuration and $240 \times 416 \times 544$ supervoxels for the $45^{\circ}$ configuration is used for DVC analyses. The positions of the ROIs in the global coordinate system are shown in Figure 1 while in the $z$ direction the ROIs and samples mid-thickness planes coincide.

The basic principle of DVC [2, 7, 49] consists of registering the gray levels $f$ in the reference and $g$ in the deformed configuration such that their conservation is obtained

$$
f(\mathbf{x})=g[\mathbf{x}+\mathbf{u}(\mathbf{x})]
$$

where $\mathbf{u}$ is the displacement field with respect to the reference volume. Due to the different noise sources in the experiments [28] the strict conservation of gray levels is never perfectly satisfied meaning the solution consists in minimizing the gray level residual $\rho(\mathbf{x})=f(\mathbf{x})-g[\mathbf{x}+\mathbf{u}(\mathbf{x})]$ over the whole ROI (i.e., global approach to DVC) by considering its L2-norm with respect 
to parameterized kinematic unknowns. The displacement field is written as

$$
\mathbf{u}(\mathbf{x})=\sum_{p} u_{p} \Psi_{p}(\mathbf{x})
$$

where $\boldsymbol{\Psi}_{p}(\mathbf{x})$ are the chosen shape functions for the parameterization of $\mathbf{u}(\mathbf{x})$. Hexahedral finite elements with trilinear shape functions are chosen in this work [38]. The global residual $\Phi^{2}$

$$
\Phi^{2}(\{\mathbf{u}\})=\sum_{\mathrm{ROI}}\left(f(\mathbf{x})-g\left(\mathbf{x}+\sum_{p} u_{p} \boldsymbol{\Psi}_{p}(\mathbf{x})\right)\right)
$$

is minimized with respect to the unknown amplitudes $u_{p}$, which are gathered in the column vector $\{\mathbf{u}\}$. This nonlinear least squares minimization is performed with a Gauss-Newton scheme [38]. After successive linearizations and corrections the current system to be solved reads

$$
[\mathbf{M}]\{\delta \mathbf{u}\}=\{\mathbf{b}\}
$$

where

$$
M_{i j}=\sum_{R O I}\left(\boldsymbol{\nabla} f \cdot \boldsymbol{\Psi}_{i}\right)(\mathbf{x})\left(\boldsymbol{\nabla} f \cdot \boldsymbol{\Psi}_{j}\right)(\mathbf{x})
$$

represents the DVC matrix, while vector $\{\mathbf{b}\}$

$$
b_{i}=\sum_{R O I}(f(\mathbf{x})-\tilde{g}(\mathbf{x}))\left(\boldsymbol{\nabla} f \cdot \mathbf{\Psi}_{i}\right)(\mathbf{x})
$$

contains the current difference between the reference volume $f$ and the corrected deformed volume $\tilde{g}=g(\mathbf{x}+\tilde{\mathbf{u}}(\mathbf{x}))$, $\tilde{\mathbf{u}}$ being the current estimate of the displacement field. The global residual needs to decrease so that convergence is achieved, i.e., the displacement corrections $\{\delta \mathbf{u}\}$ become vanishingly 
small. All the results reported hereafter require the DVC analyses to have converged, namely, the root mean square (RMS) of the displacement increment between two iterations is less than $10^{-4}$ voxel.

\subsubsection{Definition of strains and uncertainty}

From the mean gradient of the displacement field over each 8-noded element the Hencky (logarithmic) strain tensor and subsequently its second invariant will be used in the sequel, the von Mises equivalent strain being

$$
\epsilon_{e q}=\sqrt{\frac{2}{3} \boldsymbol{\epsilon}_{d}: \boldsymbol{\epsilon}_{d}}
$$

where $\boldsymbol{\epsilon}_{d}$ denotes the deviator of the logarithmic strain tensor $\boldsymbol{\epsilon}$.

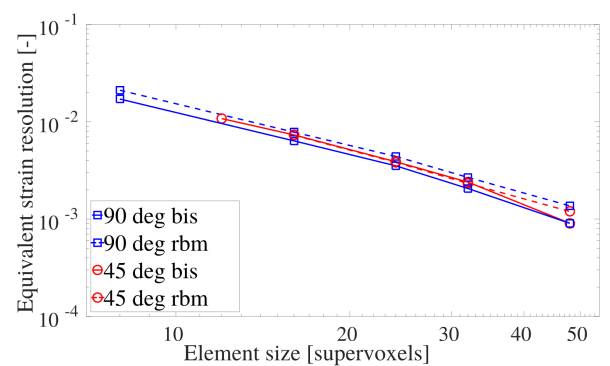

(a)

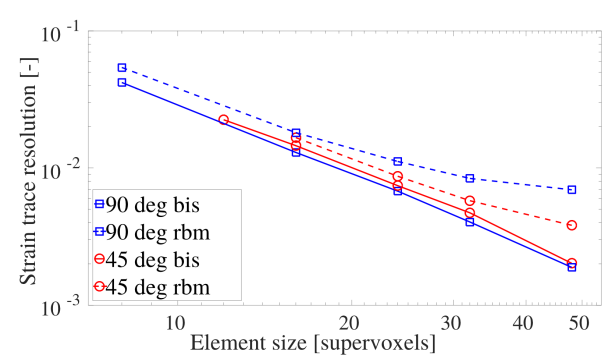

(b)

Figure 3: Equivalent strain resolution (a), and strain trace resolution (b), as functions of the element size

The DVC resolution is evaluated by correlating two scans of the unloaded sample with (denoted "rbm" in Figure 3) and without (denoted "bis" in Figure 3) a small rigid body motion applied between the acquisitions. Due to noise contributions and reconstruction artifacts, these two volumes are 
not identical. Therefore, the measured displacement field accounts for the cumulated effects of laminographic imaging and DVC on the measurement uncertainty $[28,10]$. The uncertainty values are evaluated by the standard deviation of the registered strain fields. Figure 3 shows the standard strain resolution levels for different element sizes $\ell$ expressed in supervoxels (with respect to the original voxel size). Decreasing the element size is followed by an increase of the strain uncertainty. The element size used in this work is $\ell=16$ supervoxels (length) for all three directions, which yields a standard equivalent strain resolution of $0.78 \%$ for the $90^{\circ}$ configuration and $0.73 \%$ for the $45^{\circ}$ configuration. Since the trace of the strain tensor will be considered in the following, the corresponding standard resolution for the $90^{\circ}$ configuration is $1.81 \%$ while the $45^{\circ}$ configuration has uncertainty of $1.67 \%$. These values represent the limit above which the estimated strains are deemed trustworthy.

\subsubsection{Direct vs. incremental DVC calculations}

Direct DVC results refer to the solutions obtained by correlating the undeformed volume (state (0)) and one of the deformed configurations (state $(n))$, while incremental calculations stand for the correlation between any deformed volume (state $(n))$ and subsequent $($ state $(n+1))$ configuration. Due to the large cumulated deformations the convergence of direct DVC calculations is hardly achieved for ultimate loading steps, hence an incremental correlation procedure is also followed. When displaying incremental 
calculation results the fields are shown in the reference configuration of each incremental analysis. Therefore, special care has to be exercised to identify common features to make the comparison objective. Since the global coordinate system is identical for direct and incremental calculations, the incremental displacement fields (from the end of the loading history) can be interpolated on the deformed mesh from the last converged direct calculation. By conducting such analysis one can extend DVC registrations from the undeformed configuration to the very end of the loading history. This operation is the basic principle of a cumulation procedure in postprocessing of the DVC results and will be used in the sequel [30].

\section{Results}

The mesoscale in the context of this work refers to the scale of the ligament between the two machined holes while the microscale stands for the scale of the ligaments between two nodule holes.

\subsection{Ductile damage mechanisms}

\subsubsection{Laminography observations}

Figure 4 shows the mid-thickness section of reconstructed laminography data for the two configurations during the loading history [34, 35]. For both samples the final coalescence occurred in the ligament between the machined holes. On the mesoscale, the $90^{\circ}$ configuration shows internal necking while in the $45^{\circ}$ configuration void sheeting prevails. However, one of the general 
ideas for this work is to study whether there are any differences in ductile damage mechanisms at the microscale between $45^{\circ}$ and $90^{\circ}$ cases. 

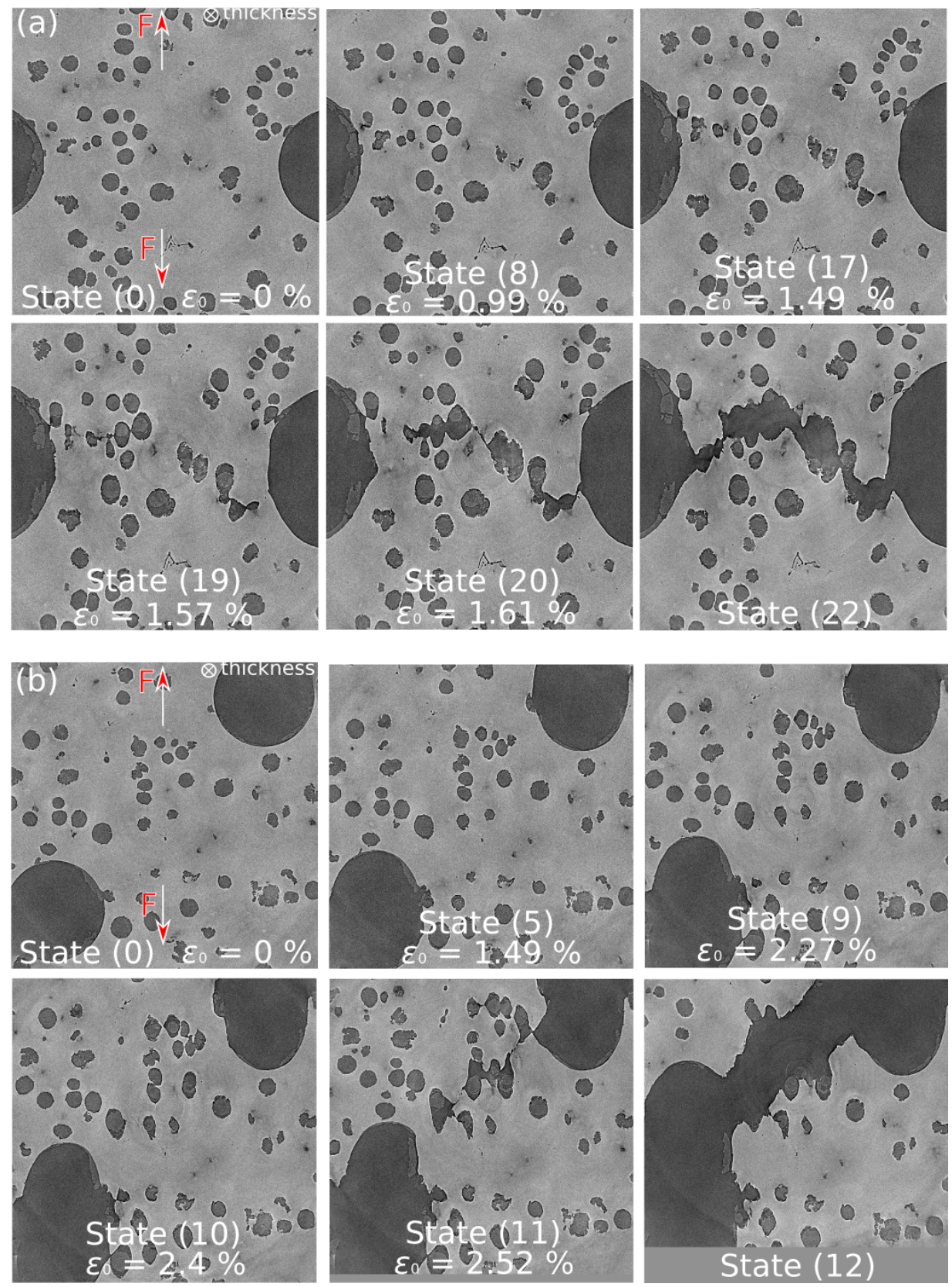

Figure 4: Mid-thickness microstructure sections of reconstructed laminography data for the $90^{\circ}$ (a) and $45^{\circ}$ (b) configurations during the loading history (The engineering strain is given as crosshead displacement divided by the gauge length). 
As a result of previous plastic flow, in the $45^{\circ}$ configuration the voids grow until fast $45^{\circ}$ macro-coalescence in the very last loading step. This macro-coalescence event mainly consists of void sheeting coalescence in the ligaments between the voids from graphite nodules that often have grown until this point under a plasticity mode involving high triaxiality.

In the $90^{\circ}$ configuration it seems that the influence of the second population (nodules) on the crack path is more pronounced in the sense that there is no regular macro-coalescence event (which here should occur normal to the loading direction connecting the two machined holes) but the final failure propagation is influenced by the graphite nodule positions. Overall, this interplay results in numerous locations (along the thickness direction $z$ ) where the final crack has a roof-top shape at the mesoscale. This type of macro-coalescence also often consists of local void sheeting events between the voids (nodules) but the prevailing mechanism is internal necking between them.

\subsubsection{DVC residuals observations: nodules and ferritic matrix}

Both samples experience early nodule debonding from the ferritic matrix. This mechanism is illustrated in Figure 5 by observing the final gray level residual $\rho(\boldsymbol{x})$. The residuals are a useful tool for precise detection of damage occurrence in cast iron [47]. Let us recall that the corrected volumes are updated at each iteration on a sub-voxel scale by using gray level interpolation (in this work a trilinear interpolation scheme has been employed). 
Therefore, by using residual fields one benefits from sub-voxel resolutions in the gray level registration procedure. All deviations from $C_{0}$ continuity such as nodule/matrix debonding, void nucleation or growth cannot be properly captured by the underlying kinematic basis. Consequently, the hypothesis of gray level conservation is no longer satisfied, which will be reflected in local residual increases. Figure 5(a) shows residual maps for the section normal to the $y$-direction for load step (0)-(5) for the $45^{\circ}$ configuration. The residual map is shown in overlay with the corresponding microstructure in the background. Note that areas of higher residuals correspond to the positions of the debond zones around nodules. The irregularly shaped nodule (denoted with a white arrow) acts as spot with concentrated residuals, i.e., specifically expressed local debonding, probably caused by high stress concentrations around this spot. Beside debond zones with higher gray level residuals, the residual map in the rest of the ROI shows sufficiently low values indicating successfully converged DVC calculations. 

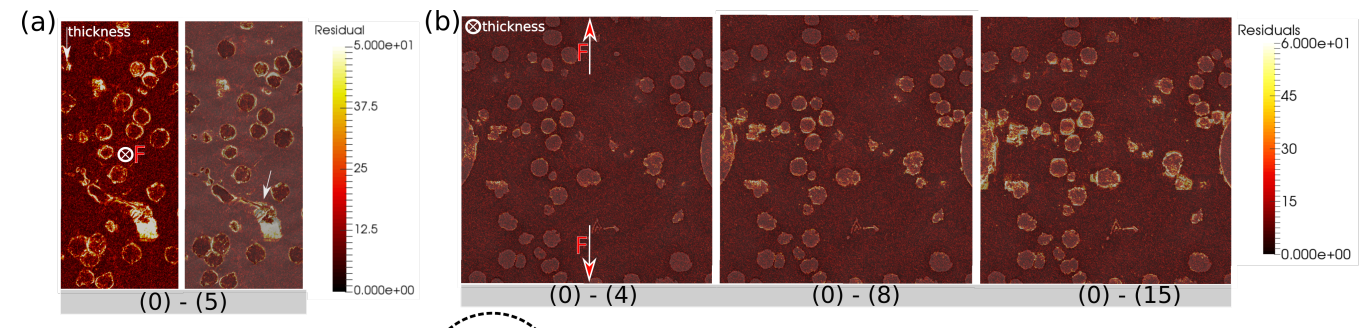

(c)

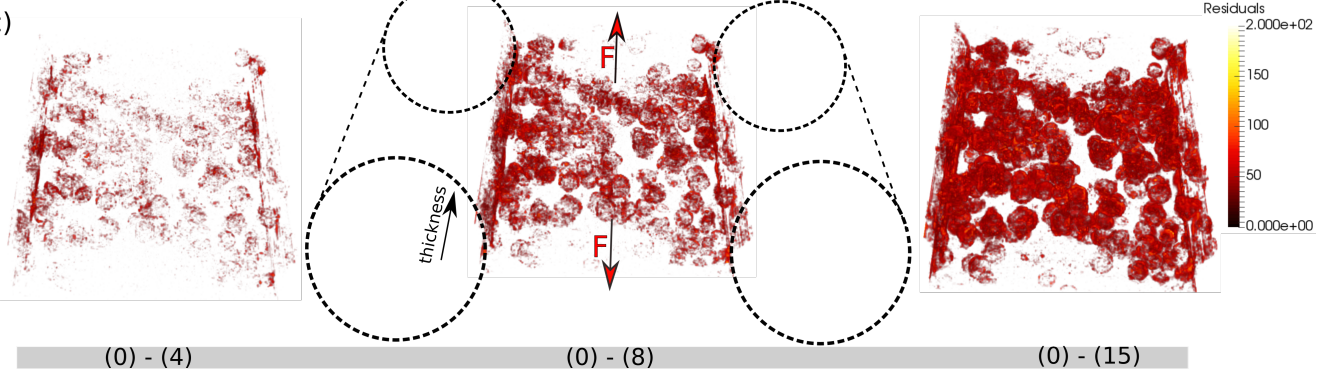

Figure 5: DVC gray level residual fields: (a) $45^{\circ}$ configuration section normal to the loading direction $y$; (b) $90^{\circ}$ configuration section normal to the thickness direction $z$; (c) isometric view of the residual field for the $90^{\circ}$ configuration

In Figure 5(b) residual field maps are shown with microstructure sections (see Figure 4) for the $90^{\circ}$ configuration. Note early debonding from load step (0)-(4) onward. Upon further loading, the residuals mainly cumulate in the ligament between the two machined holes with their peak values along the micro-localization band as shown in Figure 8 that will be commented in the sequel.

A 3D representation of the residual fields is illustrated in Figure 5(c) where its changes are observed in isometric view with a slightly inclined $z$ axis of the sample toward the observer. The residual fields are from the $90^{\circ}$ configuration. It is explicitly confirmed that most of the residual source is from debond nodules in the part of the ROI lying between the two machined 
holes.

\subsubsection{SEM fractography}

There are three distinct coalescence mechanisms found in post mortem observations on fracture surfaces: a) complete internal necking; b) incomplete necking and c) large void sheeting surfaces. Complete necking designates void growth from debond nodules characterized by high plasticity levels that occur on free void surfaces. It is marked with smooth fractured surface spots without additional small voids in the ligaments. When the distance between the nodules is sufficient they do not coalesce completely by a high stress triaxiality mechanism but void sheeting occurs at the very end, which is marked with an additional small void population (no small particles were found inside these voids) resulting in uncomplete necking. At the end, larger surface spots with small void populations are found between the machined holes and some of the nodules when the distance between the two is significant. These mechanisms can be found in both hole configurations. They are functions of the stress state but also of morphology/topology context (i.e., distance between the nodules itself, nodules and machined holes, nodule shapes and orientations).

Figure 6(a) shows the complete ligament surface for the $45^{\circ}$ configuration together with two enlarged regions. In Region A one detail is depicted and additionally enlarged in Zoom C. They are representative of incomplete internal necking (void impingement) mechanisms. Figure 6(b) displays the 
fractured surface of the $90^{\circ}$ configuration. Region D denotes the large void sheeting surface positioned between the machined hole and some of the nodules. Zoom F represents still incomplete necking since there are marks of additional nucleation occurring during final ligament coalescence. On the other side, Zoom G shows the complete internal necking mechanism, i.e., without any additional nucleation in the ligament between the nodules. The fully sheared spots (i.e., without any voids, with final shear surface alignment) are not found herein. 

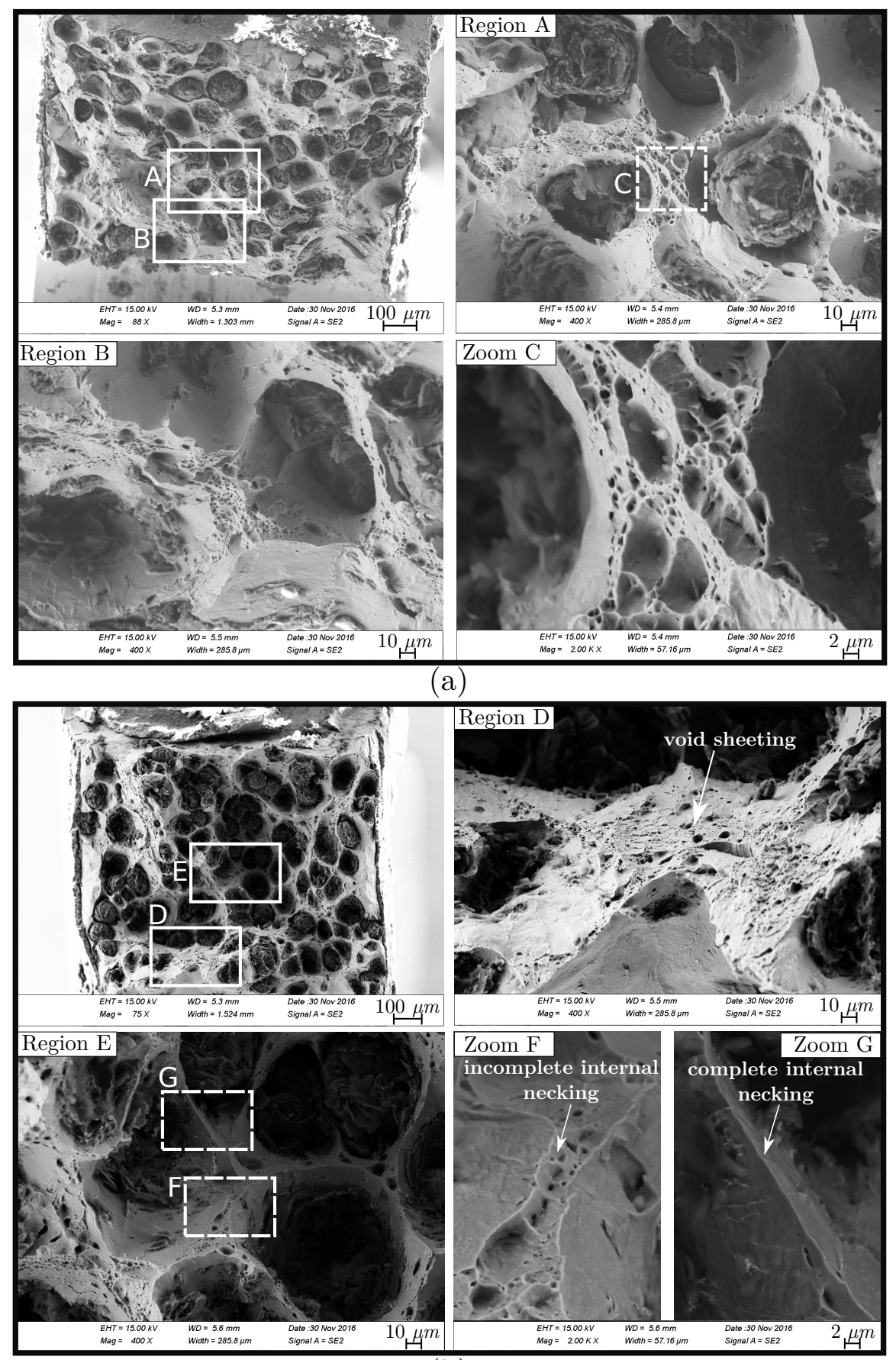

(b)

Figure 6: SEM observations for the $45^{\circ}$ (a) and $90^{\circ}$ (b) configurations 
Interestingly, even under high magnifications, no particles have been found in the pores belonging to the void sheeting zone of the fractured surface. If there are any small particles inside, it can be well recognized on the fracture surface as shown in Figure 7 (marked with green arrow). Although the objective particle is very small in size, the corresponding spot is marked with significantly higher void growth and associated plastic deformation. Hence, if there are any particles, i.e., stress concentrators present, they are easily distinguished from the empty ones among the void sheeting population. In this example most of the void sheeting population remains small in size and no particles can be found inside them meaning they probably do not nucleate on the particles but rather as outcome of large plastic deformations of the matrix and associated accumulated dislocations.

As a result of all previously mentioned observations, it can be stated that in $45^{\circ}$ and $90^{\circ}$ cases the underlying ductile damage micromechanisms are not fundamentally different, i.e., they fail locally in a similar manner.
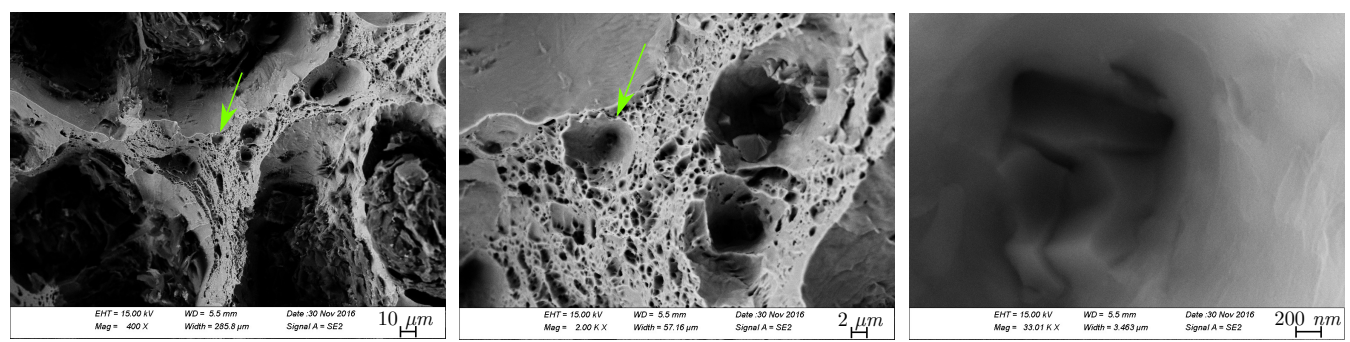

Figure 7: SEM observation of void sheeting coalescence for the $90^{\circ}$ configuration 


\subsection{Equivalent strain fields}

Figure 8 shows von Mises strain maps plotted over the corresponding microstructure sections at the sample mid-thickness. The DVC registrations could not be performed until the very end of the procedure due to (very) large deformations and significant cumulated damage (i.e., no brightness conservation). The last recorded band positions are plotted over the microstructure (yellow and red lines) to ensure better visualization up to final coalescence. 
(a)
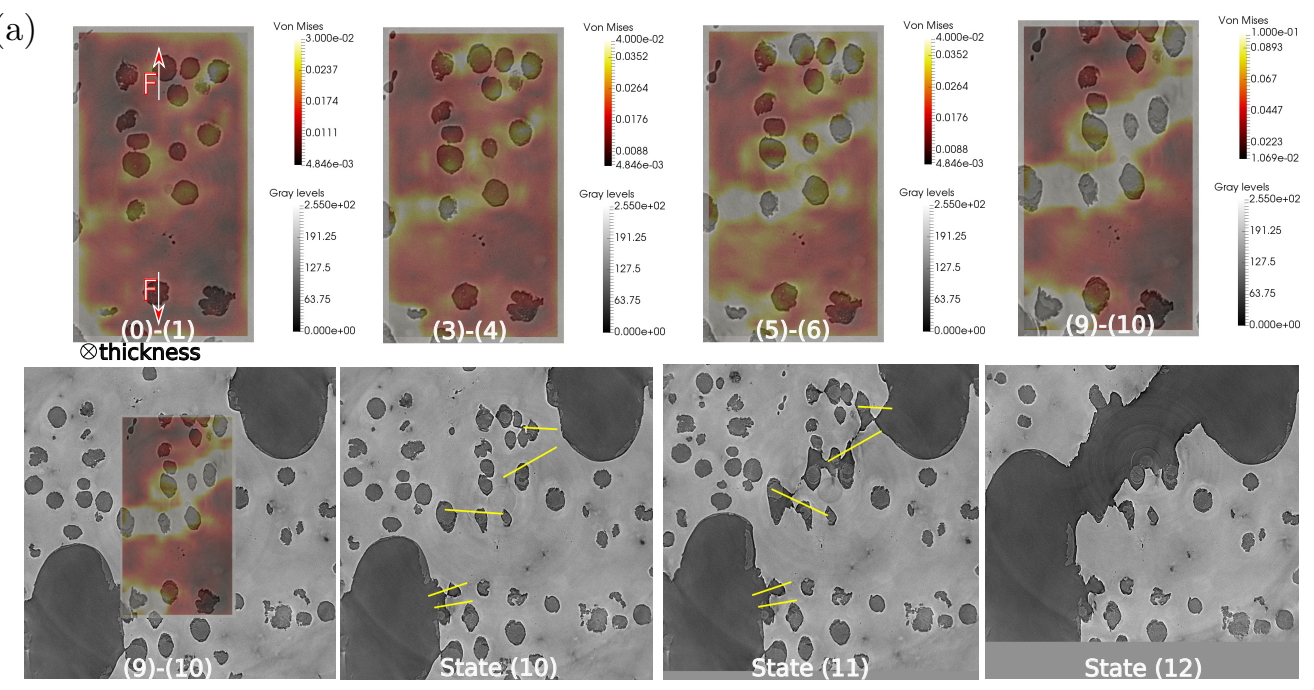

(b)
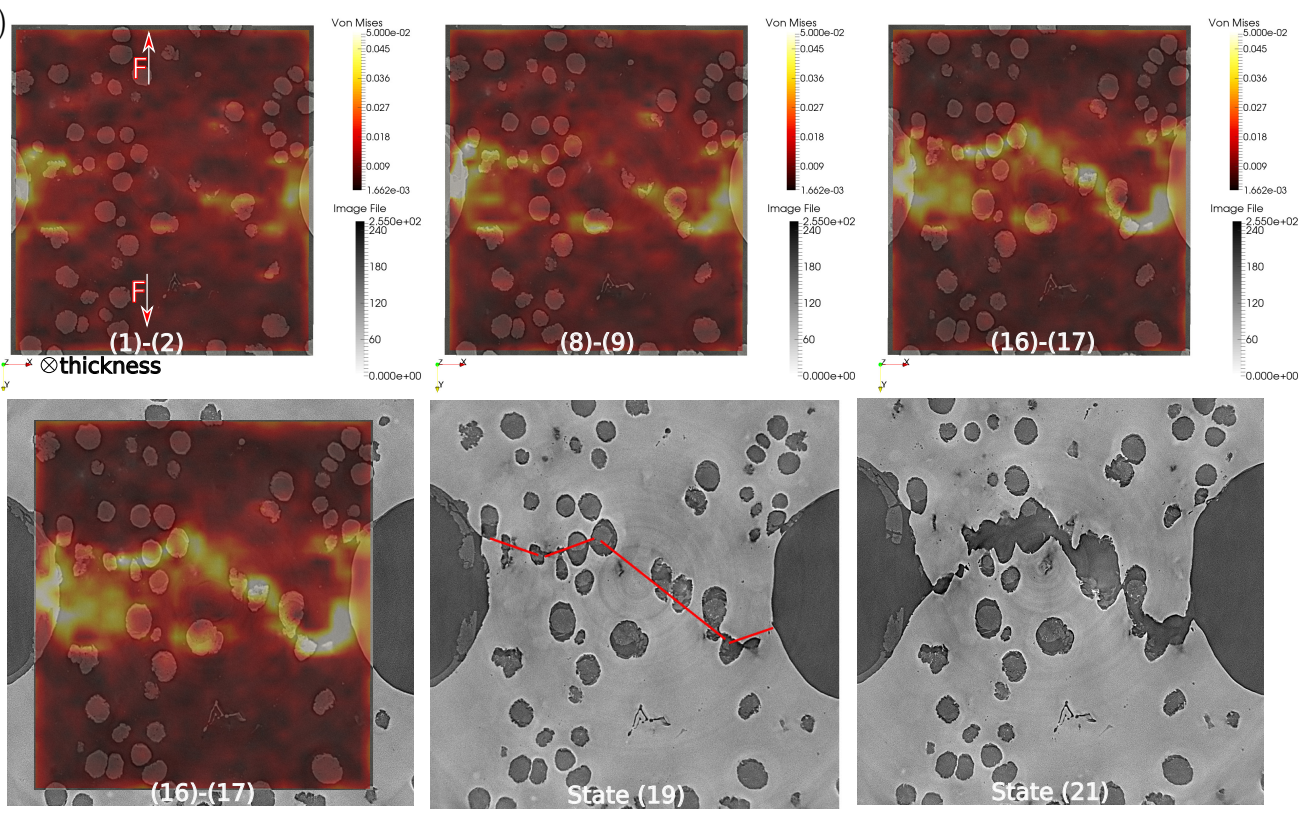

Figure 8: Von Mises strain maps plotted over the corresponding microstructure sections at the sample mid-thickness: (a) $45^{\circ}$; (b) $90^{\circ}$ configurations

From Figure $8(\mathrm{a})$ of the $45^{\circ}$ configuration early and stable strained bands are observed between the nodules. The macroband is located between the two 
machined holes, but it is rather thick (indicating non locality) and not homogeneous but determined by the local heterogeneities, i.e., nodule positions. The incremental DVC results are available up to the penultimate loading step. As a result of cumulated plastic flow, the voids develop from nodules and re-position to a favorable arrangement as precursor for fast $45^{\circ}$ coalescence between the two machined holes in the last scan. However, it is hard to judge what is the role of the nodules and the corresponding micro-strained bands (between some of them) on the final macro-coalescence event since it seems it is mainly a mesoscale effect determined by the machined hole positions and not influenced by the local nodule positions.

In the $90^{\circ}$ configuration (Figure $8(\mathrm{~b})$ ) the final failure is following more clearly the strained band positions between the nodules determined already at the beginning of the loading process. The strained bands are always containing voids, but not all of the nodules (in the area of the ligament between the machined holes) are equally affected by the band paths. Moreover some of the nodules remain unaffected.

The fact that the final failure does not follow the path of microbands in the depicted section for the $45^{\circ}$ configuration suggests that a significant amount of plastic deformation has been dissipated without direct contribution to the final coalescence propagation. Hence, the process zone is significantly wider than the crack plane itself. However, large amount of plastic deformation decreases the material hardening rate inside the ligament areas between the nodules making it easier for macro-coalescence to occur in such a sudden 
manner for the $45^{\circ}$ configuration.

\subsubsection{D analysis}

While explaining the discrepancy between the microbands and the final failure trajectory one should keep in mind that this study deals with a 3D effect and the microstructure overview along the thickness directions $z$ can help one to better understand the previous observations, especially in the $45^{\circ}$ configuration as shown in Figure 9. In particular, clusters of nodules favorably positioned are found in the neighborhood (on both sides) of the mid-thickness slice shown in Figure 8(a). In other words, these nodules are located roughly at $45^{\circ}$ already from the undeformed state. Figure 9 (state 10) reveals mesoscale void sheeting events before final macro-coalescence has occurred. It is very likely that this cluster affects the other locations where the microbands are not correlated with the final failure propagation path (see Figure 8(a)). 


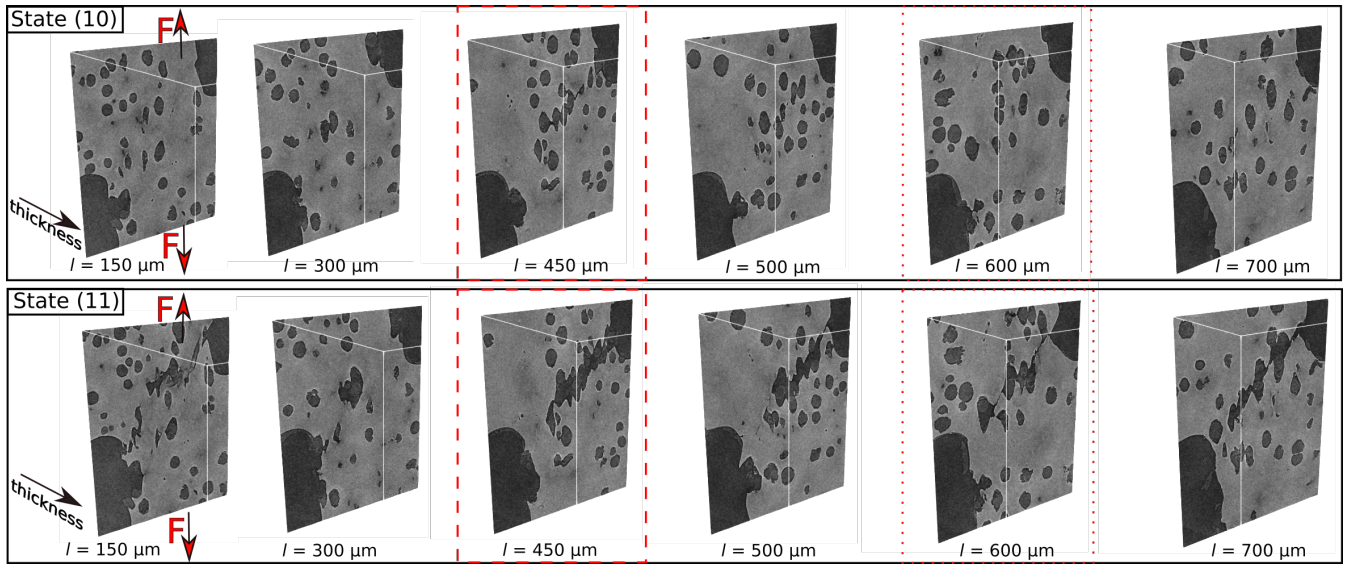

Figure 9: Microstructure sections at different positions along the thickness direction $z$ for two distinct loading steps of the $45^{\circ}$ configuration. $l$ denotes the distance from the reference sample surface whereas the mid-thickness section from Figure 8(a) is denoted with dotted lines. One of the sections with premature failure is marked with dashed lines

In Figure 10 von Mises strains are plotted over the corresponding microstructure section at the aforementioned favorably positioned nodule cluster. Studying plastic activity before meso-coalescence reveals general agreement between the microband positions and the final crack propagation path.

This case exemplifies the importance of taking into account the real 3D microstructure compared to periodic distributions often used in micromechanical studies, see e.g., Refs. [46, 45, 14]. Correlated to this observation, the use of DVC measured heterogeneous boundary conditions for driving micromechanical calculations shows significant improvement in both global and local errors between the simulations and experimental data [42]. 

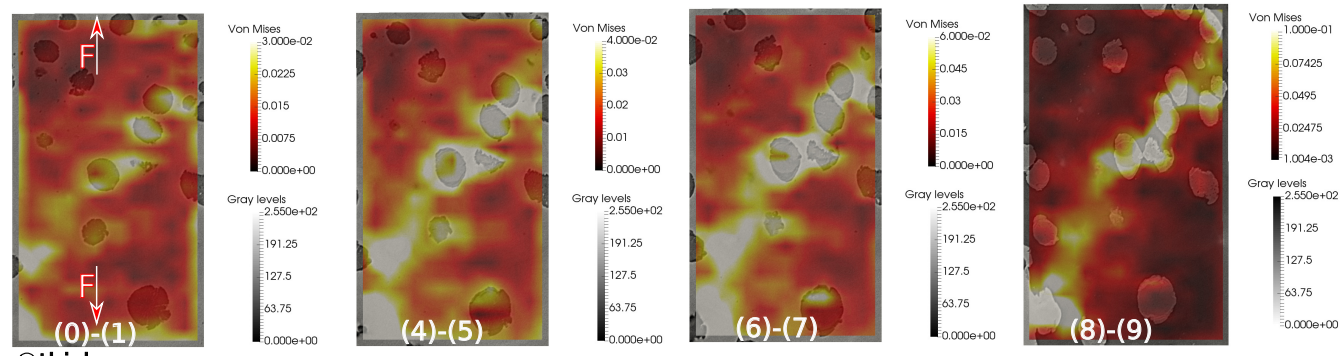

Qthickness
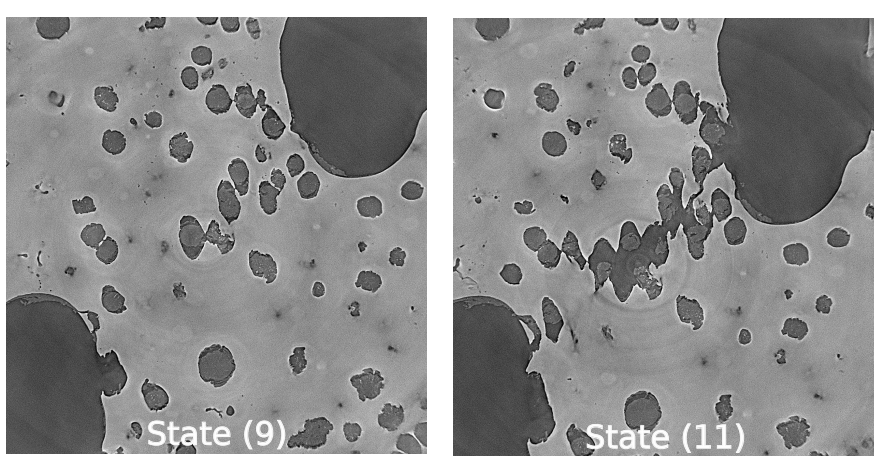

Figure 10: Von Mises strains plotted over the corresponding microstructure section with favorably positioned nodule cluster for the $45^{\circ}$ configuration. The distance from midthickness plane is $l=220 \mu \mathrm{m}$

The same analysis is repeated for the $90^{\circ}$ configuration in Figure 11. Going through the sample thickness reveals that there are numerous spots where roof-top (or V-like) shaped cracks occur. 


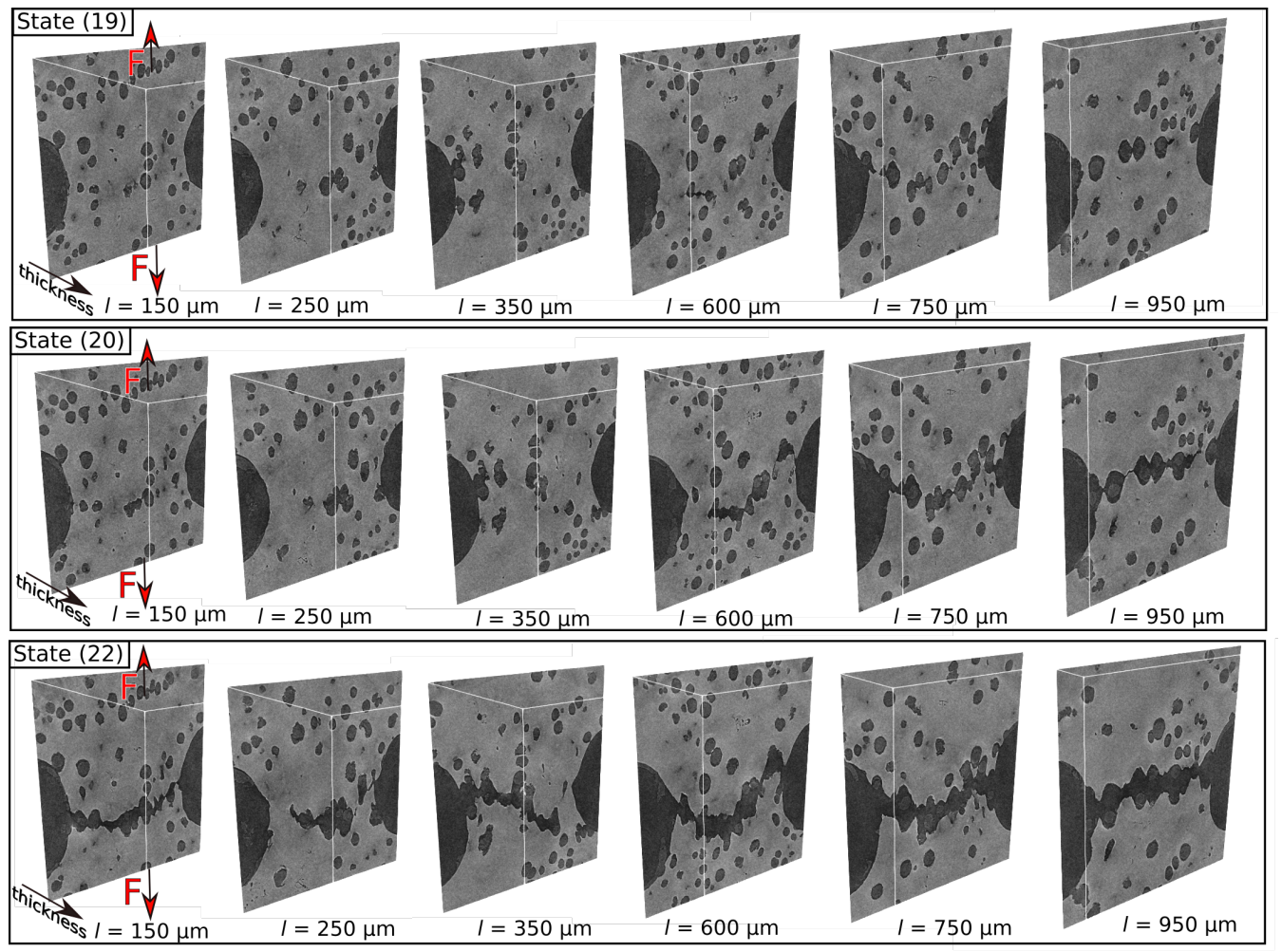

Figure 11: Microstructure sections at different positions along the thickness direction $z$ for three distinct loading steps for the $90^{\circ}$ configuration. $l$ denotes the distance from the reference sample surface

One of these spots is depicted and followed during the loading history together with the corresponding von Mises field overlays in Figure 12. It is confirmed that in the $90^{\circ}$ configuration the mesoscale strain localization effect of machined holes is less dominant, i.e., the final coalescence is rather determined by microbands between the nodules that often do not correspond to the shortest distance between the two machined holes. In this context, the $90^{\circ}$ configuration is more influenced by the $3 \mathrm{D}$ nodule distribution than 
the $45^{\circ}$ configuration for which mesoscale plastic flow governs most of the process.
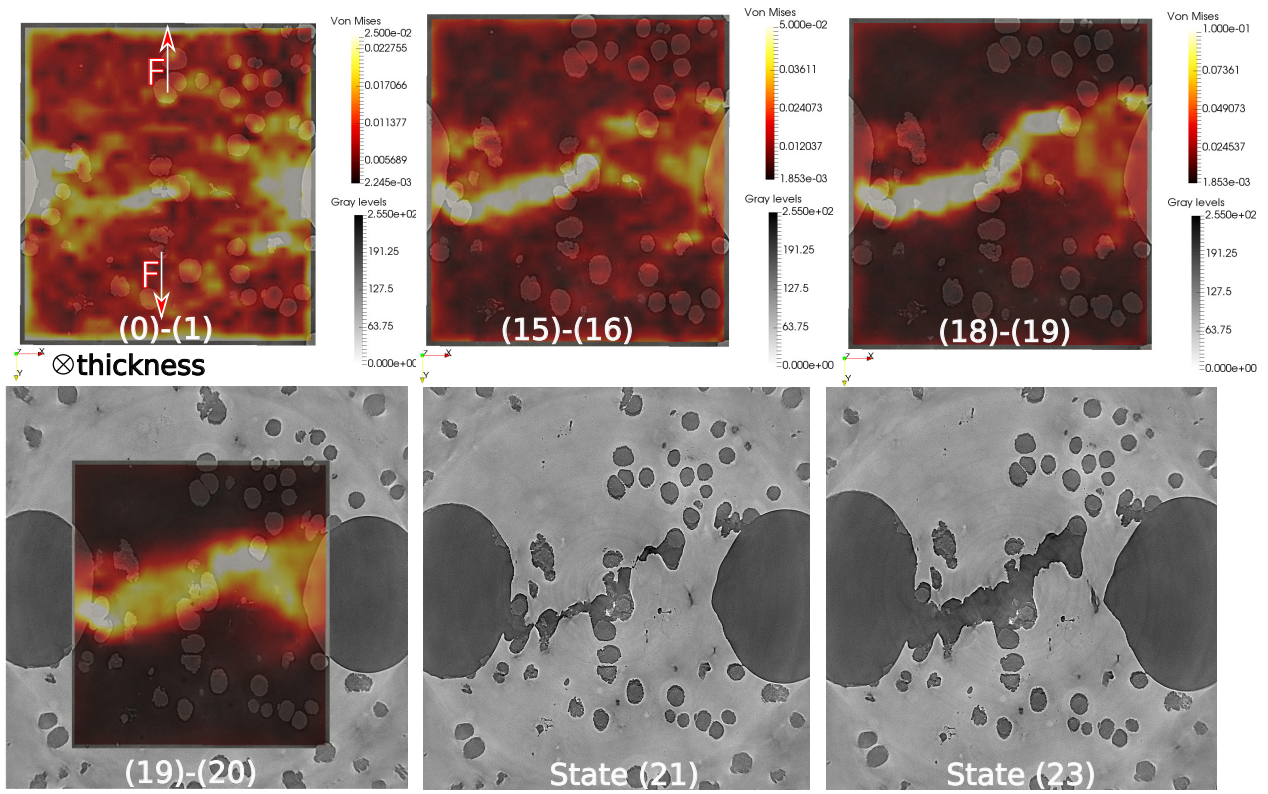

Figure 12: Von Mises strains plotted over the corresponding microstructure section for the $90^{\circ}$ configuration. The distance from mid-thickness plane is $l=275 \mu \mathrm{m}$

The effect of bulk constraints is probed in Figure 13 where the strain maps for specific loading steps are plotted over the corresponding microstructure for three different locations along the sample thickness, two of them being closer to the free-surfaces while the middle one is roughly the sample midthickness. In the $90^{\circ}$ configuration microbands spread wider when close to the surface where less constraint is present. Conversely, in the bulk the influence of the two holes is concentrating most of the plastic activity in the narrow ligament between them. 


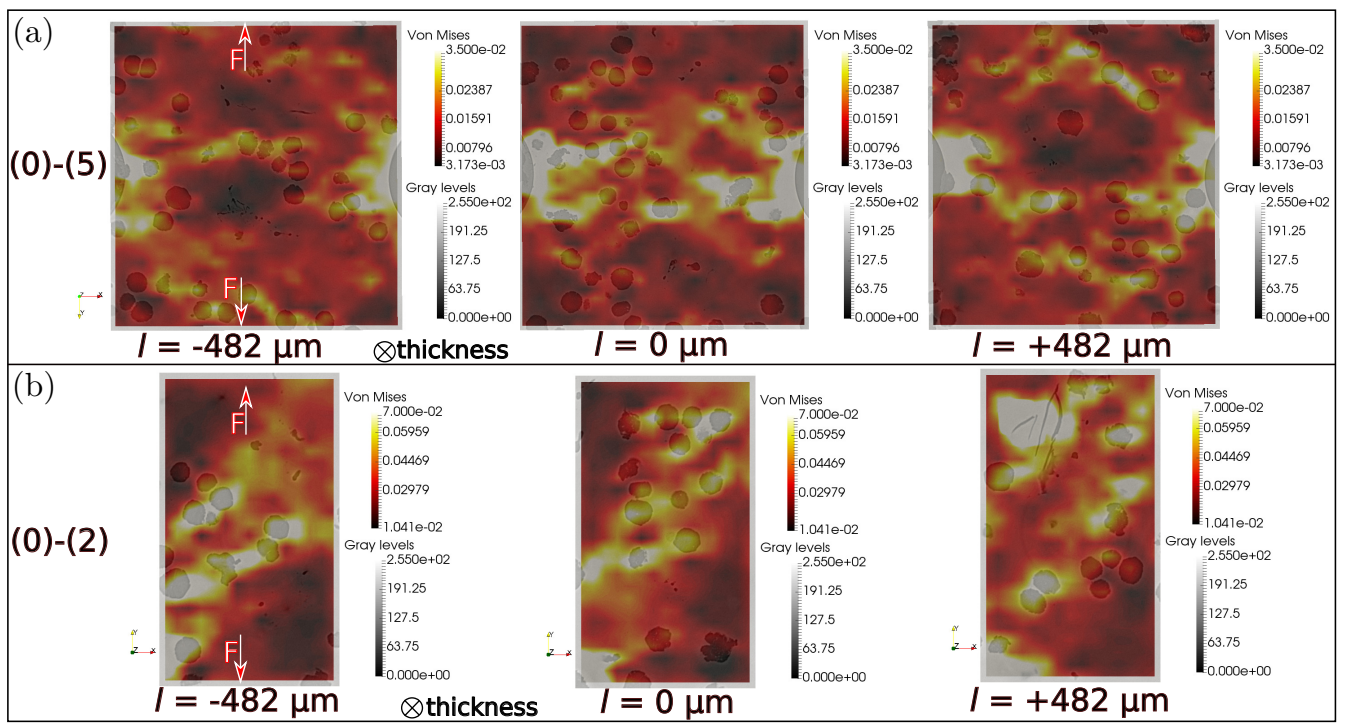

Figure 13: Von Mises strains plotted over the corresponding microstructure sections along the thickness direction $z$ for $90^{\circ}$ (a) and $45^{\circ}$ (b) configurations. $l$ denotes the distance from the ROI mid-plane in the thickness direction $z$

In the $45^{\circ}$ configuration this is not the case. The bands are strongly concentrated in the ligament irrespective of the section position.

\subsection{Quantification of the results}

In the following section the mean and local strain values are extracted from the measured kinematic fields. Those values are then correlated with the corresponding microstructure changes, i.e., void growth and coalescence effects from laminography data. Since for the late loading steps direct DVC registrations could not be conducted, incremental calculations were performed. The results from these correlations are cumulated to the latest successfully converged direct calculations. This operation results in a situation with a 
part of the boundary nodes from the initial DVC ROI without kinematic information. Hence, a priori analysis is performed and only a part of the initial ROI domain, referred to as sub-ROI (Figure 14), with available nodal displacements up to coalescence is used in the following analysis. The sub-ROIs have the same sizes, namely, $336 \times 208 \times 544$ voxels and $208 \times 336 \times 544$ voxels for the $90^{\circ}$ and $45^{\circ}$ configurations respectively.

\subsubsection{Mean kinematic values on sub-ROI level}

In Figure 14 the relationship between the nominal machine displacement and the mean von Mises strain (mean over the sub-ROI) are reported for the analyzed sub-ROIs of the two samples. The graph is accompanied with microstructure sections for important experimental instants. The last reported values are those immediately preceding overall coalescence. They will be denoted as coalescence strains in the sequel.

The coalescence strain level for the $45^{\circ}$ configuration and $90^{\circ}$ configuration are similar. This finding is different from that in Ref. [51] for $\mathrm{Al}$ alloys, which may be attributed to material effects. Standard deviations are added to the analysis. The standard deviation gives a measure of the strain heterogeneities in the sub-ROI. The fact that there is almost a linear relationship between the mean and standard deviation values reveals the stable nature of the heterogeneity of the strain fields. 

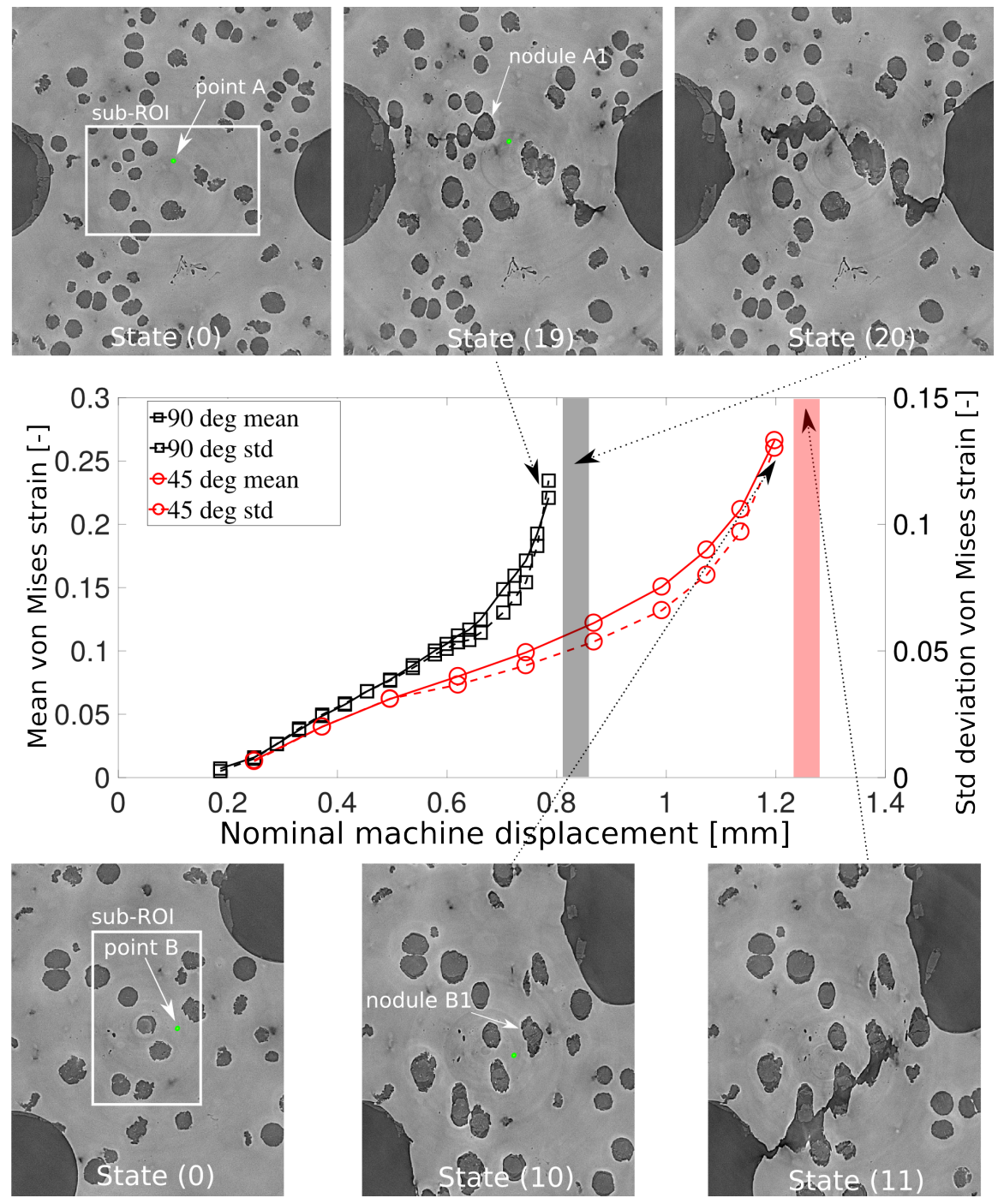

Figure 14: Mean equivalent strains from sub-ROI versus nominal machine displacement for the two samples are denoted with solid lines. The right axis shows standard deviations marked with dashed lines. Each marker stands for the scan taken during the experiment. Microstructure sections are also shown for the important experimental moments that are associated with the kinematic measurements

Figures 15 and 16 show different mean strain components as functions 
of the mean equivalent strain on sub-ROI level. Plotting the values for the normal strains in Figure 15 reveals similar slopes for $\epsilon_{y y}$ in both cases. On the other hand, $\epsilon_{x x}$ is higher for the $45^{\circ}$ configuration while the $90^{\circ}$ configuration has higher normal strains in the thickness direction $z$.

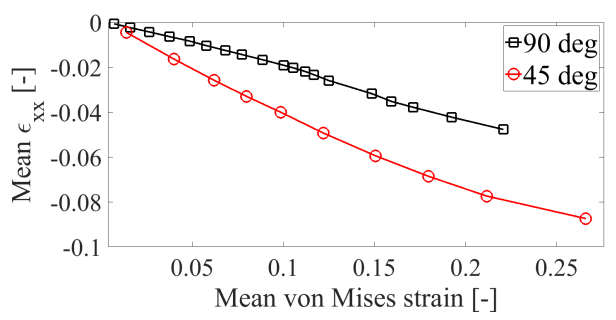

(a)

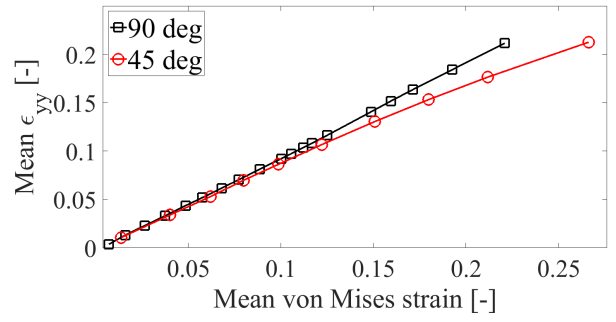

(b)

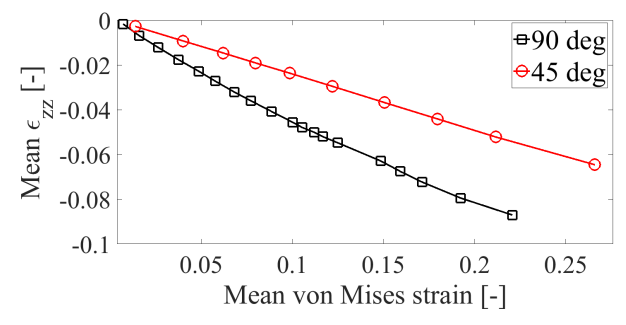

(c)

Figure 15: Mean normal strains $\epsilon_{i i}(i=x, y, z)$ plotted against the mean von Mises strains for the $90^{\circ}$ and $45^{\circ}$ configurations

The mean shear strain components $\epsilon_{i j}(i=x, y, z$ and $j=y, z, x)$ are depicted in Figure 16. It clearly illustrates the expected dominance of $\epsilon_{x y}$ component in the $45^{\circ}$ configuration over all other shear components for both samples. The final values $(>0.1)$ for $\epsilon_{x y}$ outline the importance of the shear component knowing it accounts for more than $50 \%$ of the normal strain component in the loading direction for the $45^{\circ}$ configuration. 


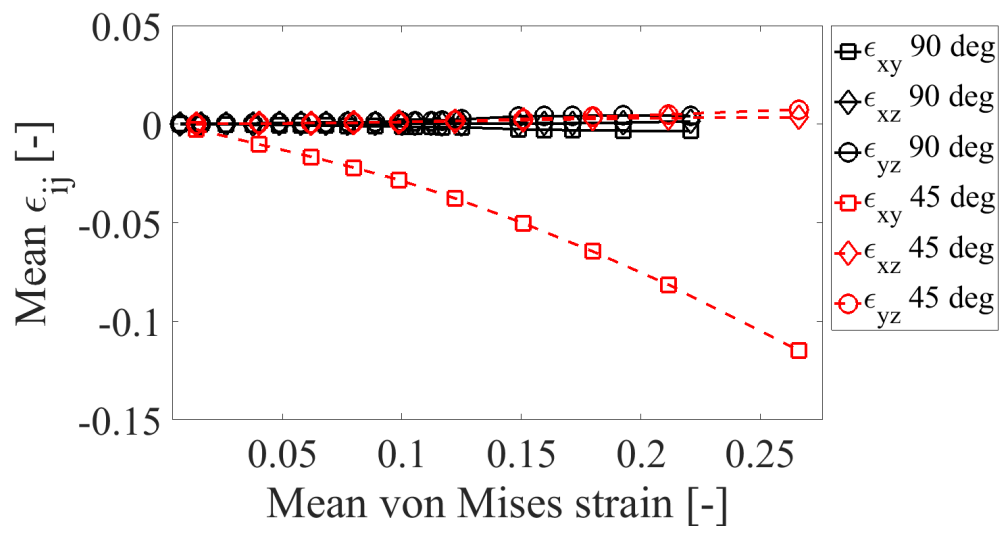

Figure 16: Shear strains $\epsilon_{i j}(i, j=x, y, z)$ plotted against the mean von Mises strain for both configurations

Both samples experience a positive and stable strain trace from the beginning of the loading process. In the second part of loading history, for the same levels of mean von Mises strains, the $90^{\circ}$ configuration experiences higher strain traces, which indicates higher void growth $\left(\sum \epsilon_{i i}=0\right.$ for constant volume). This result is depicted in Figure 17(a).

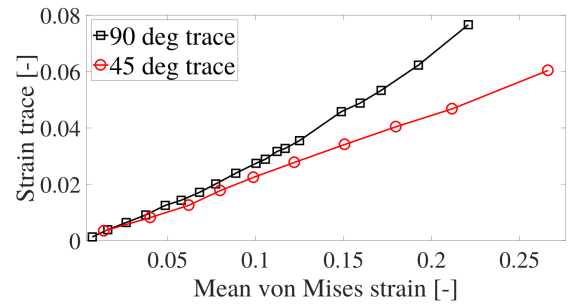

(a)

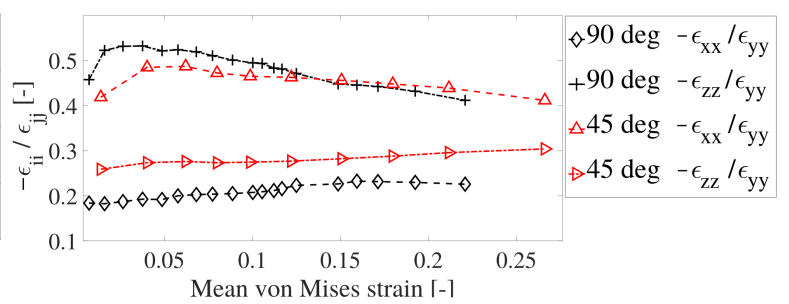

(b)

Figure 17: Mean strain trace (a) and negative ratio (b) between the normal strains $\epsilon_{x x}$, $\epsilon_{z z}$ and the normal strains in the stretching direction $\epsilon_{y y}$ plotted as functions of the mean equivalent von Mises strains for the two configurations 
The ratios between the normal strains $\epsilon_{x x}, \epsilon_{z z}$ and the normal strains in the stretching direction $\epsilon_{y y}$ are shown in Figure $17(\mathrm{~b})$. The $90^{\circ}$ configuration experiences stronger necking along the thickness direction. This can be explained by the wider macroband shown in Figures 13 .

Conversely, the $45^{\circ}$ configuration shows significant relative necking in the $x$-direction. In the first part of the loading history $\epsilon_{x x} / \epsilon_{y y}$ is close to 0.5 indicating there is low constraint in the $x$-direction due to the machined holes. However, combined with the low $\epsilon_{z z} / \epsilon_{y y}$ ratio it results in non-isochoric deformation with positive trace from the beginning of loading, i.e., increasing volume. Although the normal strain ratios vary significantly during loading, the sum of the two ratios is relatively stable and amounts to $\approx 0.74$ for both configurations.

However, this result does not imply a plane strain condition along the thickness direction in the $45^{\circ}$ configuration as shown in Figure 18 where the normal strains $\epsilon_{z z}$ in the thickness direction are plotted for three different positions along the ligament between the holes for the two samples. Both $90^{\circ}$ and $45^{\circ}$ configurations exhibit (mostly) contracted zones and some zones around nodules with higher resistance to lateral deformation. This kind of localized straining between nodules is present from the early loading steps on. However, it differs from those reported for Al-alloys in slanted regions of thin Compact Tension-like samples as the localization between nodules can be reproduced by von Mises plasticity [9]. For Al-alloy CT-like specimens, in contrast, a plane strain condition along the crack propagation direction 
were identified and the localization is hard to be predicted in models using von Mises plasticity [27, 8].

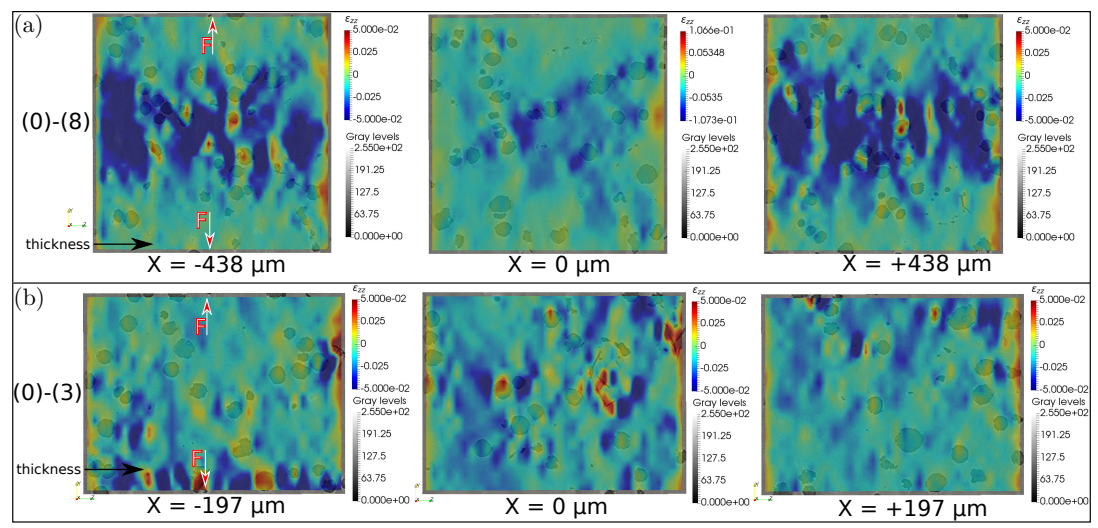

Figure 18: Normal strains $\epsilon_{z z}$ plotted over the corresponding microstructure sections for three different positions along the ligament between the holes for $90^{\circ}$ (a) and $45^{\circ}$ (b) configurations. $X$ denotes the distance from ROI mid plane in $x$ direction

Figure 19 reveals that there are normal strains $\epsilon_{x x}$ in the crack propagation direction for both $90^{\circ}$ and $45^{\circ}$ configurations. Nodular graphite cast iron represents another class of material compared to $\mathrm{Al}$ alloys and straining between the nodules is mainly a structural effect. Yet, it is confirmed herein that the plane strain condition is not a necessary condition for very early and stable straining. 


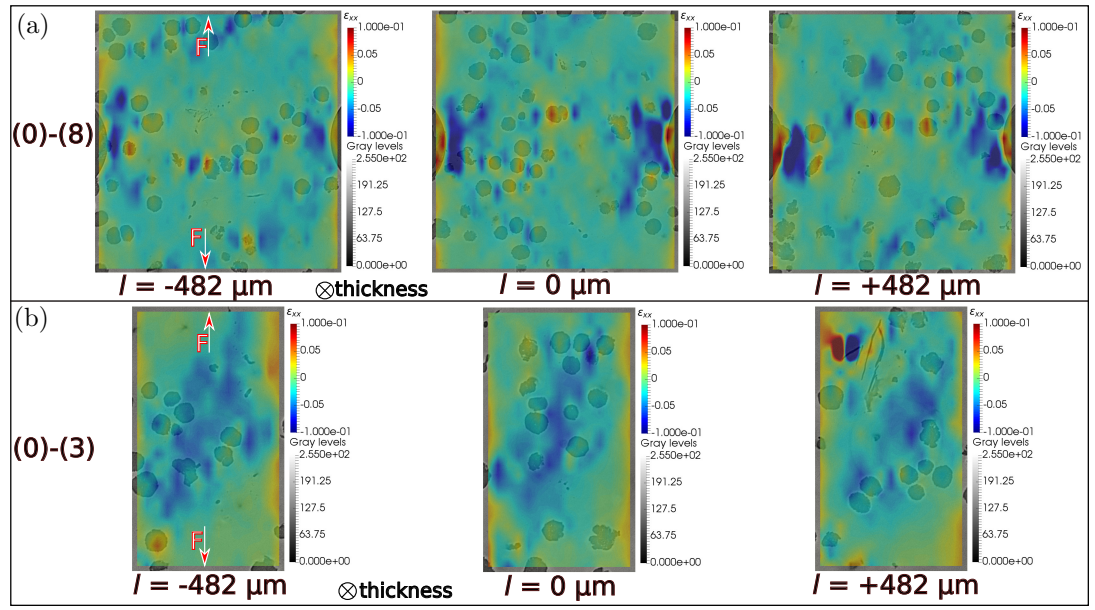

Figure 19: Normal strains $\epsilon_{x x}$ plotted over the corresponding microstructure sections for three different positions along the ligament between the holes for $90^{\circ}$ (a) and $45^{\circ}$ (b) configurations. $l$ denotes the distance from the ROI mid-plane in the thickness direction $z$

\subsubsection{Local strain levels between nodules}

In Figure 20 local von Mises strains are plotted against the corresponding mean values. The positions of points of interest for local values are depicted in Figures 14 and 20 with yellow dots. The chosen points A and B lie in the ligaments that later experience similar void sheeting between the nodules (Figure 14). The local coalescence equivalent strains (solid lines) have similar values for the cumulated plastic strain prior to ligament failure for both configurations. In this example, for locally sheared zones, the cumulated von Mises strain proved to be adequate for the description of the material ductility and a threshold value of $\approx 40 \%$ for the onset of failure is found in both configurations. 


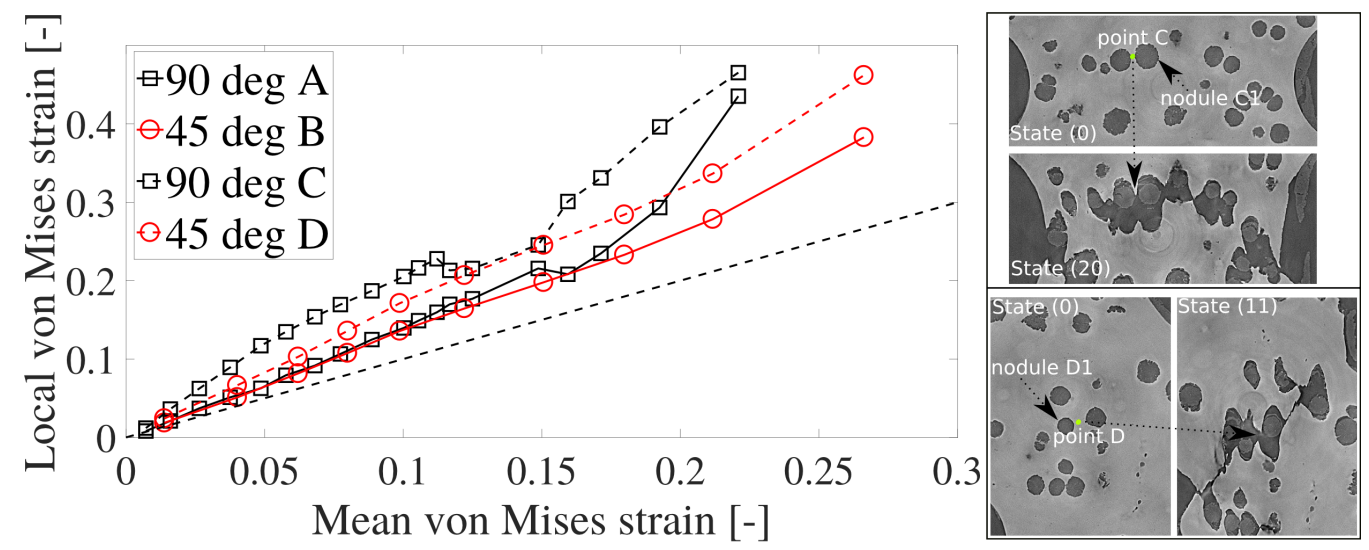

Figure 20: Local values (inter-nodule) of von Mises strain for the points of interest denoted on the right $(\mathrm{C}, \mathrm{D})$ and in Figure 14 (A, B) plotted against the mean von Mises strains in the corresponding sub-ROIs. The dashed line without markers denotes one-to-one relationship for more convenient reading of the graph

In Figure 20 points $\mathrm{C}$ and D (i.e., representatives of internal necking ligaments) are also analyzed. Again, similar values for the coalescence equivalent strains for the two samples (dashed lines) are found $(\approx 45 \%)$. Note that these levels are close to those obtained in the void sheeting ligaments and seem to be independent of the ligament failure type. In other words, this result underlines the ability of equivalent von Mises strains to locally indicate material failure. However, it remains insensitive to the type of damage mechanisms and final ligament failure, i.e., these items did not reflect in von Mises strains changes (i.e., the slope of the curves for different mechanisms are similar) nor its final values. It is the local nodule arrangement of the real microstructure that is of prime importance, then the nodule shapes and finally matrix defects that will induce different stress states responsible for 
different ligament failures.

The local strain trace levels exhibit some sensitivity to different ligament failure mechanisms (Figure 21). For each of the configurations, the two different failure mechanisms can now be distinguished due to the different strain trace levels and ultimate values. This is especially true for the $45^{\circ}$ configuration. Although they fail in a similar manner as shown in Figure 14 the strain trace levels for points A and B differ substantially. The same observation is valid for points $\mathrm{C}$ and $\mathrm{D}$ meaning that the local trace cannot be used as global threshold quantity for different meso-stress states, but when limited to the defined state (in this case $90^{\circ}$ or $45^{\circ}$ configuration) it is a helpful physical quantity for the distinction of the two ligament failure mechanisms.

This example also illustrates the importance of proper scale modeling since on the ligament level both sample geometries failed in a similar manner as already shown in post mortem analyses but not with an identical kinematic history (e.g., different strain trace levels). The real added value of this analysis to the SEM images is the possibility of following the bulk kinematics/microstructure changes during the whole experiment. 


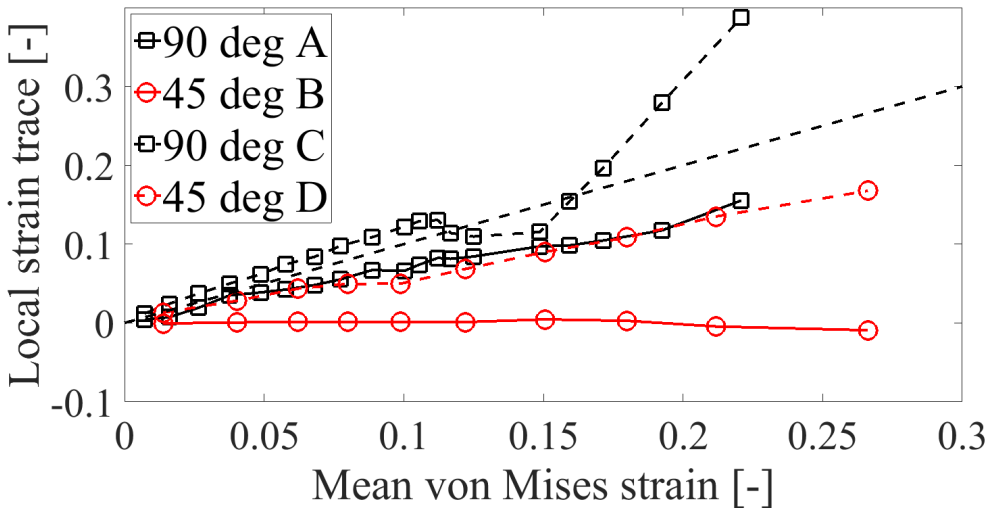

Figure 21: Local strain (inter-nodule) trace values for the points of interest depicted in Figures 14 and 20 plotted against the mean von Mises strain levels in the corresponding sub-ROIs

\subsection{Void growth vs. kinematic data}

\subsubsection{Void growth in the ROI}

The common way of quantifying the void volume change/damage growth is by segmenting gray level images and following the number of remaining voxels that normally should belong to voids [24]. The analysis based on this approach is performed in this work but with certain improvements. First, by means of DVC measured kinematic fields the initial set of material points is followed throughout the experiment (i.e., a truly Lagrangian measurement). Second, the good choice for gray level threshold, which is often subjective, is probed by employing the measured mean strain trace. Void growth obtained with different thresholds from laminography data $(\delta f)$ is compared with the volume change calculated by the mean strain trace multiplied by the current 
sub-ROI volume $(\delta V)$. The results are shown in Figure 22 for the two analyzed samples. The best agreement is obtained for a threshold of 115 gray levels for both samples.

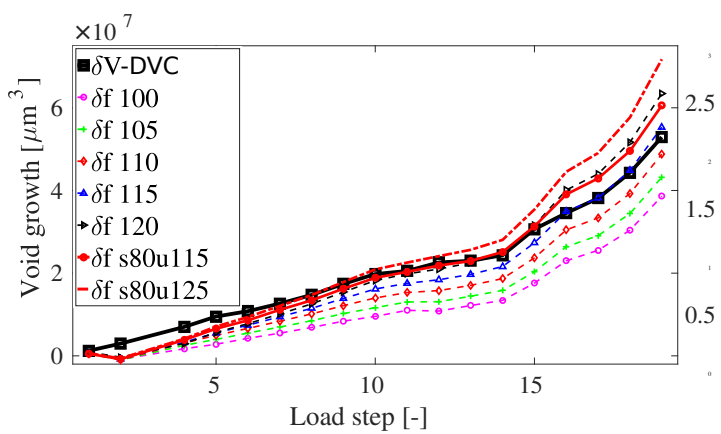

(a)

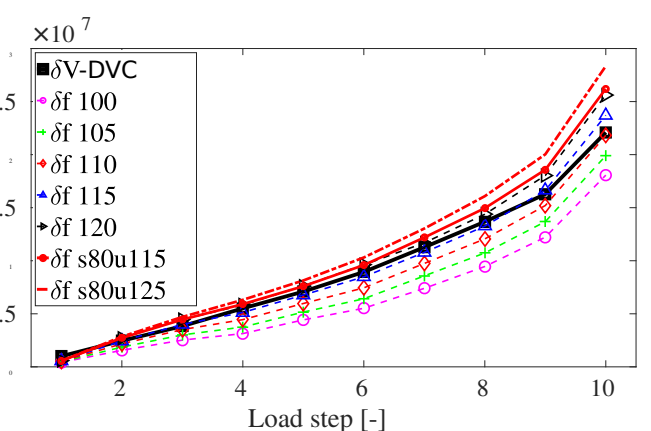

(b)

Figure 22: Void growth obtained with different threshold values from laminography data $(\delta f)$ expressed as total volume increase in the ROI compared with the volume change $\delta V$ calculated by the mean strain trace multiplication with the current ROI volume $(\delta V)$ for the $90^{\circ}$ (a) and $45^{\circ}$ (b) configurations

However, if one performs more sophisticated procedures while creating binary images that include several operations from image analysis procedure, namely, placing seeds on nodule positions followed by limited threshold growth and fill hole operations on binary images two additional curves are obtained and marked with thicker red lines in Figure 22 (denoted as $\delta f s 80 u 115$ and $\delta f s 80 u 125)$. When compared to $\delta V$ values from the DVC analysis the gap is somewhat higher than that obtained with $\delta f 115$ curve. The reason for this effect is probably due to the fill hole operation in Fiji software [39, 41, 40] whose effect is illustrated in Figure 23. Again this operation prevents the 
attempts of better calibration of this threshold method to $\delta V$ values since the fill hole step compensates the reduction of the threshold. Nonetheless, the latter method for thresholding has physically the best sense as shown in Figure 23 and the values for $\delta f s 80 u 115$ from Figure 22 will be used in the sequel. Kinematic data (strain trace) are thus shown to be a useful tool for the initial estimation of the proper gray level values for thresholding methods when calculating void growth from tomography/laminography data.

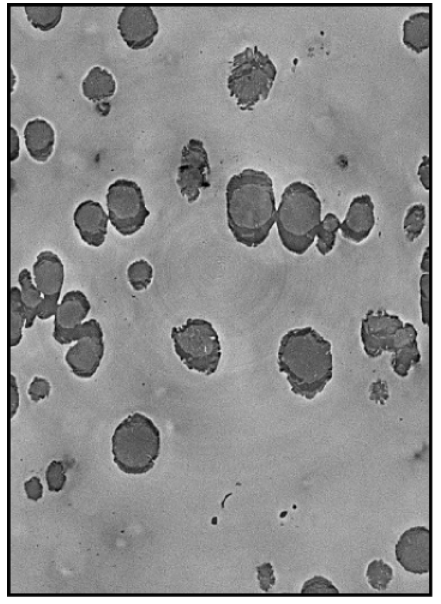

microstructure section

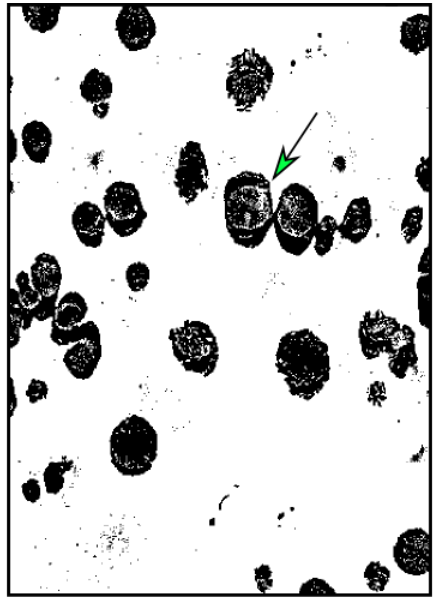

threshold $\mathrm{GL}=115$

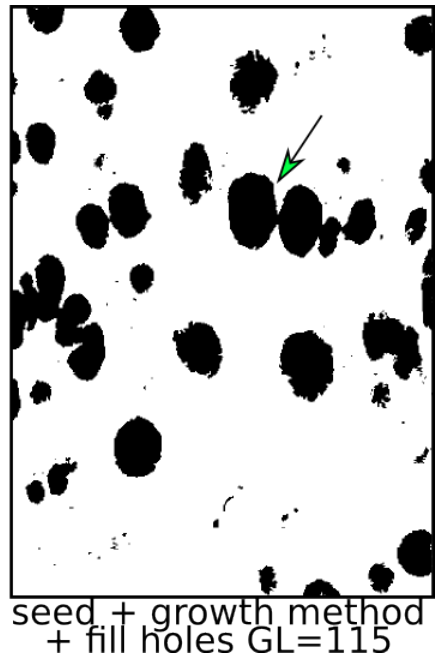

Figure 23: Comparison of two different threshold methods applied in this work. The voxels counted as voids are colored in black

Figure 24 displays the void volume fraction in the ROI as a function of the mean equivalent strain. The void volume fraction is obtained by division with the current ROI volume during the experiment. Despite the strong shear strain component for the $45^{\circ}$ configuration, the void volume fractions prior to coalescence are similar for both cases, namely, $\approx 16 \%$. 


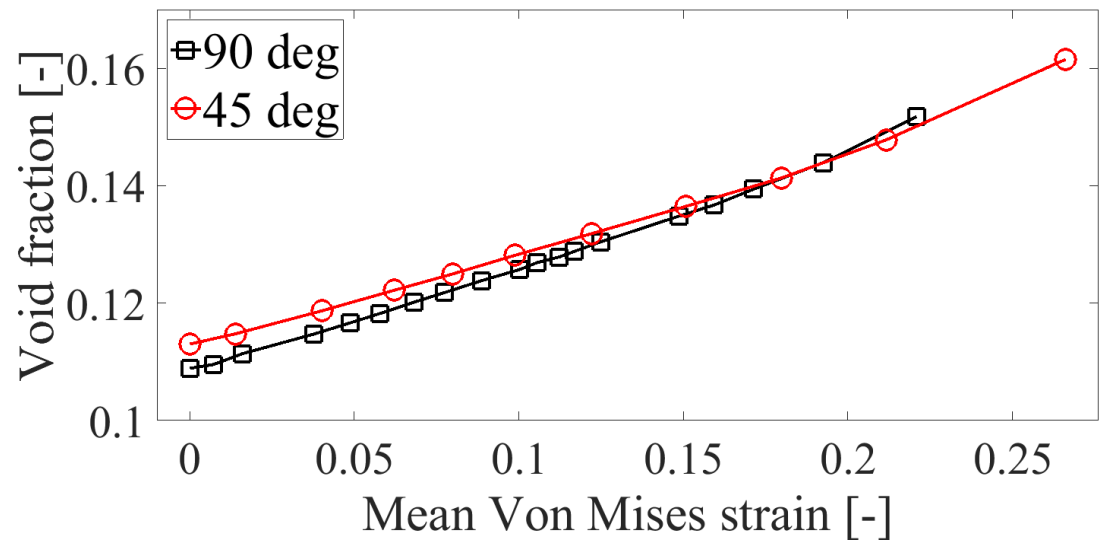

Figure 24: Void volume fraction versus mean von Mises strain on the level of sub-ROI for the two configurations

\subsubsection{Local values}

For the local scale analysis between void growth and kinematic data the following approach is applied. Void growth associated with the 4 nodules positioned in the immediate vicinity of points A, B (void sheeting ligaments from Figure 14) and C, D (internal necking ligaments from Figure 20) is followed during the experiment. The positions of the nodules are also depicted in Figures 14 and 20. Their designations are associated with the corresponding points of interest, hence A1, B1, C1, D1. The volumes of the nodule+void (due to early debonding and growth) are normalized by the corresponding initial nodule volume. The same procedure is repeated with the surfaces of the voids. The counting is performed by Fiji software [5, 39, 41, 40] and the results are shown in Figure 25. Note that even on the local scale where both ligaments experience a similar type of failure void growth is still higher for 
the $90^{\circ}$ configuration. No significant deviations from this conclusion have been observed for the void surface changes.

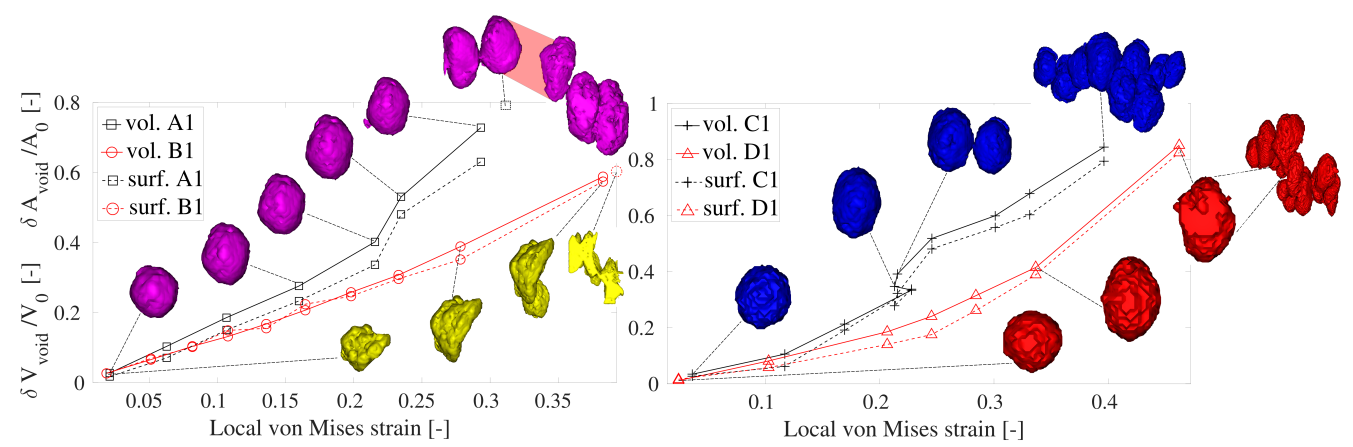

Figure 25: Volume (solid) and surface (dashed) growth of voids from nodules A1, B1, C1 and D1 normalized with their initial volumes/surfaces plotted against the corresponding local von Mises strains for points A, B, C and D (see Figure 14 and 20 for positions of the points)

On the left side of Figure 25, voids for $90^{\circ}$ (A1) and $45^{\circ}$ (B1) configurations are followed through the experiment and accompanied by void representations isolated from the original laminography volumes. The $90^{\circ}$ configuration void (magenta) experiences stable growth up to $30 \%$ of local strain when the first signs of internal necking occur on the left side of the void. In the following loading step the void is linked by internal necking with numerous surrounding voids, while many of them are further connected with their subsequent neighbors creating a significant network of linked voids. This is the reason why the measurement is not conducted here. One additional figure is added showing just part of the mentioned linked network containing a wide corridor (shaded) between the two mini-void-clusters where sheet co- 
alescence will occur in the following step. A similar scenario is observed for the $45^{\circ}$ configuration void (yellow). Just before the onset of void sheeting shown on most right image, the followed void also interacted with the closest neighbor in an internal necking linkage mode.

The right side of Figure 25 shows internal necking ligament voids for $90^{\circ}(\mathrm{C} 1)$ and $45^{\circ}$ (D1) configurations. In the $90^{\circ}$ case the void (blue) grows individually up to $22 \%$ of the measured local strain when it starts to interact with its environment. In the next loading step the void representation shows the onset of internal necking linkage between the monitored void and its neighbor. Note that point $\mathrm{C}$ is placed between these two voids (Figure 20). Since complete linkage has not occurred and only the two illustrated voids are interacting, the measurements are carried out up to the point when more general coalescence occurred as illustrated at the ultimate measured point. The $45^{\circ}$ void (red) experiences more stable growth to the very end of the measurements. Only for the last step the studied void coalesces with the group of voids and the linked network is established. The measurement is still performed but the monitored void is previously isolated from the network. The same isolation procedure is followed in all other cases where coalescence partly occurred but measurements were still reported, i.e., only the volume/surface of the controlled void is reported, not those from the whole linked assembly of voids. 


\section{Conclusions}

The effect of the arrangement of two artificial machined voids on ductile void growth and coalescence mechanisms is studied in flat specimens made of nodular graphite cast iron. The damage changes are imaged via in situ 3D laminography. Digital volume correlation is employed for bulk strain measurement. Two configurations of machined holes are studied i) $90^{\circ}$ with respect to the loading direction to mimic internal necking and ii) $45^{\circ}$ to trigger void sheeting mechanisms between the artificial voids. The machined holes are assumed to play the role of primary voids, the graphite nodules the role of secondary voids. Fractography revealed at a lower scale a smaller third population of voids.

On the mesoscale, it is shown that the $45^{\circ}$ artificial void configuration reproduced the expected shear strain band. For the coalescence mechanism the secondary voids (nucleating from nodules) played a minor role, i.e., the final crack path was not affected by the nodules. In the $90^{\circ}$ configuration, a more diffuse plastic zone between the machined holes favored the influence of secondary (nodule) voids on the crack path, namely, the final failure followed the early prescribed localization paths between nodules that, occasionly, did not represent the shortest distance between the primary voids.

On the microscale, secondary voids (from nodules) in both configurations tended to initially grow in high triaxiality mode and interact with the closest neighbors in an internal necking mode. At the very late stages of deformation just prior to the final failure void sheeting coalescence took place in some 
locations; in the $45^{\circ}$ configuration mainly because of the mesoscale strain band effect determined by the primary void positions while in the $90^{\circ}$ sample void sheeting is the outcome of the favorably positioned clusters of already linked voids that finally needed to coalesce to form the main failure crack. The SEM analyses confirmed these micromechanisms at smaller scales in both configurations (Figure 6). Nanometric dimples are seen on the fracture surface but their origin remains undetermined. The main conclusion from this work is the complexity of multiscale and strong $3 \mathrm{D}$ nodule distribution effects revealed in the two samples.

The mean equivalent strain on the level of the sub-ROI (i.e., most of the ligament between the machined holes) shows slightly higher ductility of the $45^{\circ}$ configuration. The mean trace of the strain tensor, i.e., volume change, does not differ significantly for the two cases (Figure 21), which corresponds to early debonding and void growth on the microscale.

For the microscale internodule coalescence analysis two different ligament types for both cases were depicted, namely, one of them experiences void sheeting while the other classical internal necking coalescence. Here the local von Mises strain levels prior to coalescence were substantially higher than that of the mesoscale, but they are similar for both samples (void sheeting ligaments $\approx 40 \%$; internal necking ligaments $\approx 45 \%$, see Figure 20 ). The normalized individual void growth values in the immediate vicinity of the mentioned ligaments were determined. The results, shown in Figure 25, outline that even though the ligaments will end up with the same type of 
failure, void growth of boundary voids is higher in the $90^{\circ}$ configuration for both types of coalescence mechanisms.

Last, let us note that the present data will be used in the future as input and validation data for micromechanical modeling to probe plasticity and damage models using real microstructures and measured boundary conditions [42].

\section{Acknowledgements}

The financial support of the Fédération Francilienne de Mécanique and Agence Nationale de la Recherche (ANR-14-CE07-0034-02 grant for COMINSIDE project) is gratefully acknowledged. We acknowledge the European Synchrotron Radiation Facility for provision of beamtime at beamline ID15 (experiment ME 1366) and Marco di Michiel for help during the experiment. M. Kuna and L. Zybell from IMFD, TU Freiberg, are thanked for materials supply and machining as well as for scientific discussions. F. Gaslain CdM, MINES ParisTech is thanked for the SEM analyses. 


\section{References}

[1] Y. Bai and T. Wierzbicki. Application of extended mohr-coulomb criterion to ductile fracture. International Journal of Fracture, 161(1):1-20, 2010 .

[2] B. Bay, T. Smith, D. Fyhrie and M. Saad. Digital volume correlation: three-dimensional strain mapping using X-ray tomography. Experimental Mechanics, 39:217-226, 1999.

[3] A. Benzerga, D. Surovik and S. Keralavarma. On the path-dependence of the fracture locus in ductile materials analysis. International Journal of Plasticity, 37:157-170, 2012.

[4] J. Besson. Continuum models of ductile fracture: A review. International Journal of Damage Mechanics, 19:3-52, 2010.

[5] S. Bolte and F. P. Cordelières. A guided tour into subcellular colocalization analysis in light microscopy. Journal of Microscopy, 224(3):213-232, 2006.

[6] N. Bonora and A. Ruggiero. Micromechanical modeling of ductile cast iron incorporating damage. Part I: Ferritic ductile cast iron. International Journal of Solids and Structures, 42(5-6):1401-1424, 2005.

[7] M. Bornert, J. Chaix, P. Doumalin, J. Dupré, T. Fournel, D. Jeulin, E. Maire, M. Moreaud and H. Moulinec. Mesure tridimensionnelle de 
champs cinématiques par imagerie volumique pour l'analyse des matériaux et des structures. Instrumentation, Mesure, Métrologie, 4:43-88, 2004.

[8] A. Buljac, T. Taillandier-Thomas, T. F. Morgeneyer, L. Helfen, S. Roux and F. Hild. Slant strained band development during flat to slant crack transition in AA 2198 T8 sheet: in situ 3D measurements. International Journal of Fracture, 200(1):49-62, 2016.

[9] A. Buljac, M. Shakoor, J. Neggers, L. Helfen, M. Bernacki, P.-O. Bouchard, Thilo F. Morgeneyer and F. Hild. Numerical Validation Framework for Micromechanical Simulations based on Synchrotron 3D Imaging. Computational Mechanics, 59(3):419-441, 2017.

[10] A. Buljac, T. Taillandier-Thomas, L. Helfen, T.F. Morgeneyer and F. Hild. Evaluation of measurement uncertainties of digital volume correlation applied to laminography data. The Journal of Strain Analysis for Engineering Design, in press (DOI:10.1177/0309324717748097), 2017.

[11] D. J. Bull, L. Helfen, I. Sinclair, S. M. Spearing and T. Baumbach. A Synthesis of Multi-Scale 3D X-ray Tomographic Inspection Techniques for Assessing Carbon Fibre Composite Impact Damage. Composites Science and Technology, 75:55-61, 2013.

[12] T.-S. Cao, C. Bobadilla, P. Montmitonnet and P.-O. Bouchard. A comparative study of three ductile damage approaches for fracture prediction 
in cold forming processes. Journal of Materials Processing Technology, 216:385-404, 2015.

[13] T.-S. Cao, E. Maire, C. Verdu, C. Bobadilla, P. Lasne, P. Montmitonnet and P.-O. Bouchard. Characterization of ductile damage for a high carbon steel using 3D X-ray micro-tomography and mechanical tests Application to the identification of a shear modified GTN model. Computational Materials Science, 84:175-187, 2014.

[14] K. Danas and P. Ponte Castañeda. Influence of the Lode parameter and the stress triaxiality on the failure of elasto-plastic porous materials. International Journal of Solids and Structures, 49(11-12):1325-1342, 2012.

[15] M. J. Dong, C. Prioul and D. François. Damage effect on the fracture toughness of nodular cast iron: Part I. Damage characterization and plastic flow stress modeling. Metallurgical and Materials Transactions A, 28(11):2245-2254, 1997.

[16] M. Dunand and D. Mohr. On the predictive capabilities of the shear modified Gurson and the modified Mohr Coulomb fracture models over a wide range of stress triaxialities and Lode angles. Journal of the Mechanics and Physics of Solids, 59(7):1374-1394, 2011.

[17] A. L. Gurson. Continuum theory of ductile rupture by void nucleation and growth: Part I - yield criterion and flow rules for porous ductile 
media. ASME Journal of Engineering Materials and Technology, 99:215, 1977.

[18] L. Helfen, T.F. Morgeneyer, F. Xu, M. N. Mavrogordato, I. Sinclair, B. Schillinger and T. Baumbach. Synchrotron and neutron laminography for three-dimensional imaging of devices and flat material specimens. International Journal of Materials Research, 2012(2):170-173, 2012.

[19] L. Helfen, A. Myagotin, P. Pernot, M. DiMichiel, P. Mikulík, A. Berthold and T. Baumbach. Investigation of hybrid pixel detector arrays by synchrotron-radiation imaging. Nuclear Instruments and Methods in Physics Research Section B, 563:163-166, 2006.

[20] G. Hütter, L. Zybell and M. Kuna. Micromechanisms of fracture in nodular cast iron: From experimental findings towards modeling strategies - A review. Engineering Fracture Mechanics, 144:118-141, 2015.

[21] C. Landron, E. Maire, J. Adrien, H. Suhonen, P. Cloetens and O. Bouaziz. Non-destructive 3-D reconstruction of the martensitic phase in a dual-phase steel using synchrotron holotomography. Scripta Materialia, 66(12):1077-1080, 2012.

[22] J. Lorthios, F. Nguyen, A.-F. Gourgues, T. F. Morgeneyer and P. Cugy. Damage observation in a high-manganese austenitic TWIP steel by synchrotron radiation computed tomography. Scripta Materialia, 63(12):1220-1223, 2010. 
[23] E. Maire and P. J. Withers. Quantitative X-ray tomography. International Materials Reviews, 59(1):1-43, 2014.

[24] E. Maire, S. Zhou, J. Adrien and M. Dimichiel. Damage quantification in aluminium alloys using in situ tensile tests in x-ray tomography. Engineering Fracture Mechanics, 78:2679-2690, 2011.

[25] V. Maurel, L. Helfen, F. N’Guyen, A. Köster, M. Di Michiel, T. Baumbach and T. F. Morgeneyer. Three-dimensional investigation of thermal barrier coatings by synchrotron-radiation computed laminography. Scripta Materialia, 66:471-474, 2012.

[26] T. F. Morgeneyer, L. Helfen, I. Sinclair, H. Proudhon, F. Xu and T. Baumbach. Ductile crack initiation and propagation assessed via in situ synchrotron radiation computed laminography. Scripta Materialia, 65:1010-1013, 2011.

[27] T. F. Morgeneyer, T. Taillandier-Thomas, L. Helfen, T. Baumbach, I. Sinclair, S. Roux and F. Hild. In situ 3D observation of early strain localisation during failure of thin Al alloy (2198) sheet. Acta Materialia, pages 78-91, 2014.

[28] T. F. Morgeneyer, L. Helfen, H. Mubarak and F. Hild. 3D Digital Volume Correlation of Synchrotron Radiation Laminography images of ductile crack initiation: An initial feasibility study. Experimental Mechanics, 53(4):543-556, 2013. 
[29] T. F. Morgeneyer, H. Proudhon, P. Cloetens, W. Ludwig, Q. Roirand, L. Laiarinandrasana and E. Maire. Nanovoid morphology and distribution in deformed HDPE studied by magnified synchrotron radiation holotomography. Polymer, 55(25):6439-6443, 2014.

[30] T. F. Morgeneyer, T. Taillandier-Thomas, A. Buljac, L. Helfen and F. Hild. On strain and damage interactions during tearing: 3D in situ measurements and simulations for a ductile alloy (AA2139-T3). Journal of the Mechanics and Physics of Solids, 96:550-571, 2016.

[31] A. Myagotin, A. Voropaev, L. Helfen, D. Hänschke, T. Baumbach. Efficient Volume Reconstruction for Parallel-Beam Computed Laminography by Filtered Backprojection on Multi-Core Clusters. IEEE Transactions on Image Processing, 22(12):5348-5361, 2013.

[32] A. Needleman and V. Tvergaard. An analysis of ductile rupture in notched bars. Journal of the Mechanics and Physics of Solids, 32(6):461490, 1984.

[33] V. Tvergaard and K. M. Nielsen. Ductile shear failure or plug failure of spot welds modelled by modified Gurson model. Engineering Fracture Mechanics, 77(7):1031-1047, 2010.

[34] J. Ahrens, B. Geveci and C. Law. ParaView: An End-User Tool for Large Data Visualization. Visualization Handbook, Elsevier ISBN-13: 978-0123875822, 2005. 
[35] U. Ayachit. The ParaView Guide: A Parallel Visualization Application. Kitware, ISBN 978-1930934306, 2015.

[36] P. Reischig, L. Helfen, A. Wallert, T. Baumbach and J. Dik. Noninvasive, three-dimensional x-ray imaging of paint layers. Applied Physics A, 111:983-995, 2013.

[37] E. Roux, M. Shakoor, M. Bernacki and P.-O. Bouchard. A new finite element approach for modelling ductile damage void nucleation and growth-analysis of loading path effect on damage mechanisms. Modelling and Simulation in Materials Science and Engineering, 22(7):075001, 2014.

[38] S. Roux, F. Hild, P. Viot and D. Bernard. Three dimensional image correlation from X-Ray computed tomography of solid foam. Composites Part A: Applied Science and Manufacturing, 39(8):1253-1265, 2008.

[39] J. Schindelin, I. Arganda-Carreras, E. Frise, V. Kaynig, M. Longair, T. Pietzsch, S. Preibisch, C. Rueden, S. Saalfeld, B. Schmid, J. Y. Tinevez, D. J. White, V. Hartenstein, K. Eliceiri, P. Tomancak and A. Cardona. Fiji: an open-source platform for biological-image analysis. Nature Methods, 9(7):676-682, 2012.

[40] J. Schindelin, C. T. Rueden, M. C. Hiner and K. W. Eliceiri. The imagej ecosystem: An open platform for biomedical image analysis. Molecular Reproduction and Development, 82(7-8):518-529, 2015. 
[41] C. A. Schneider, W. S. Rasband and K. W. Eliceiri. NIH Image to ImageJ: 25 years of image analysis. Nature Methods, 9(7):671-675, 2012.

[42] M. Shakoor, A. Buljac, J. Neggers, F. Hild, Thilo F. Morgeneyer, L. Helfen, M. Bernacki and P.-O. Bouchard. On the choice of boundary conditions for micromechanical simulations based on 3D imaging. International Journal of Solids and Structures, 112:83-96, 2017.

[43] T. Taillandier-Thomas, S. Roux, T. F. Morgeneyer, F. Hild, Localized strain field measurement on laminography data with mechanical regularization. Nuclear Instruments and Methods in Physics Research Section B, 324:70-79, 2014.

[44] C. C. Tasan, J. P. M. Hoefnagels and M. G. D. Geers. Identification of the continuum damage parameter: An experimental challenge in modeling damage evolution. Acta Materialia, 60(8):3581-3589, 2012.

[45] C. Tekog̃lu, J.-B. Leblond and T. Pardoen. A criterion for the onset of void coalescence under combined tension and shear. Journal of the Mechanics and Physics of Solids, 60(7):1363-1381, 2012.

[46] V. Tvergaard. Influence of voids on shear band instabilities under plane strain conditions. International Journal of Fracture, 17(4):389-407, 1981.

[47] Z. Tomičević, J. Kodvanj and F. Hild. Characterization of the nonlinear behavior of nodular graphite cast iron via inverse identification analysis 
of uniaxial tests. European Journal of Mechanics - A/Solids, 59:140-154, 2016.

[48] T. Ueda, L. Helfen, T. F. Morgeneyer. In-situ laminography study of three-dimensional individual void shape evolution at crack initiation and comparison with GTN-type simulations. Acta Materialia, 78C:254-270, 2014.

[49] E. Verhulp, B. van Rietbergen and R. Huiskes. A three-dimensional digital image correlation technique for strain measurements in microstructures. Journal of Biomechanics, 37(9):1313-1320, 2004.

[50] M. Vogelgesang, T. Farago, T. F. Morgeneyer, L. Helfen, T. dos Santos Rolo, A. Myagotin and T. Baumbach. Real-time image content based beamline control for smart 4D X-ray imaging. Journal of Synchrotron Radiation, 23(5):1254-1263, 2016.

[51] A. Weck and D. S. Wilkinson. Experimental investigation of void coalescence in metallic sheets containing laser drilled holes. Acta Materialia, $56(8): 1774-1784,2008$.

[52] L. Xue, J. Faleskog and J. W. Hutchinson. Tension - torsion fracture experiments - Part II: Simulations with the extended Gurson model and a ductile fracture criterion based on plastic strain. International Journal of Solids and Structures, 50(25-26):4258 - 4269, 2013. 
[53] K. S. Zhang, J. B. Bai and D. François. Ductile fracture of materials with high void volume fraction. International Journal of Solids and Structures, 36(23):3407-3425, 1999.

[54] F. Xu, L. Helfen, A. J. Moffat, G. Johnson, I. Sinclair and T. Baumbach. Synchrotron radiation computed laminography for polymer composite failure studies. Journal of Synchrotron Radiation, 17(2):222-226, 2010. 